\title{
Waveform inversions of SS precursors: An investigation of the northwestern Pacific subduction zones and intraplate volcanoes in China
}

\author{
Ramin M. H. Dokht ${ }^{\mathrm{a},{ }^{*}}$, Yu Jeffrey Gu ${ }^{\mathrm{a}}$, Mauricio D. Sacchi ${ }^{\mathrm{a}}$ \\ a Department of Physics, \\ University of Alberta, \\ Edmonton, AB, Canada, T6G2E1 \\ *Email address: ramin1@ualberta.ca (Ramin M. H. Dokht)
}

\begin{abstract}
The arrival time and amplitude of long-period underside reflection from mantle seismic discontinuities (SS precursors) have made major contributions to the understanding of mantle composition and dynamics. In this study, we introduce a nonlinear waveform inversion technique to simultaneously constrain shear velocities and discontinuity depths beneath the northwestern Pacific subduction system. Based exclusively on a large SS precursor waveform dataset, we are able to clearly delineate the morphology of the descending Pacific plate, which flattens at the base of the upper mantle and extends westward by at least $\sim 1500 \mathrm{~km}$ toward northern-central China. Our grid search over a range of angles indicates a maximum correlation between shear velocity and transition zone thickness at $\sim 30$ degrees, consistent with the reported average slab dip beneath the study region. The strongly positive correlation suggests predominantly thermal, rather than compositional, variations along the descending Pacific plate. Our joint depth-velocity solutions also suggest a 5-10 km depression of the $410 \mathrm{~km}$ discontinuity and an average decrease of $1.2 \%$ in upper mantle shear velocity beneath the intraplate volcanic fields in northeastern China. This anomaly, which reaches the middle of the upper mantle transition zone beneath the Changbai hotspot, initiates at a significantly shallower $(\sim 320 \mathrm{~km})$ depth beneath the Wudalianchi regions. The high amplitude reflection at depths greater than $410 \mathrm{~km}$ suggests a water-poor melt layer in possible association with 1) decompression melting from passive upwelling and/or 2) active upwelling through a slab window.
\end{abstract}

Keywords: SS precursor, Mantle transition zone, Northwestern Pacific, Intraplate volcanism, Waveform inversion 


\section{Introduction}

Intraplate volcanic activities have been well documented in both continental and oceanic regions at distances hundreds to thousands of kilometers away from

40 plate boundary zones. The origin and mechanism of intraplate volcanism vary 41 broadly (Niu, 2005; Zhao, 2007; Tang et al., 2014) and often require the presence 42 of deep mantle plumes (Campbell, 2007; Chen et al., 2007; Zhao, 2007). An ideal 43 laboratory for the study of intraplate volcanism is northeastern Asia, where 44 Cenozoic magmatic centers are densely distributed along the north-south oriented

45 Changbai Mountain range and Wudalianchi volcanic field in northeastern (NE) 46 China. The former is a stratovolcano located approximately $1200 \mathrm{~km}$ west of the 47 Japan trench, while the latter consists of cinder volcanoes covering an area of 500 $48 \mathrm{~km}^{2}$ toward the north. The origin of these volcanic fields has been linked to 49 mantle plumes as well as subduction-related back-arc spreading and thinning of 50 the lithosphere (Basu et al., 1991; Niu, 2005), though compatible helium isotopic 51 compositions between the Cenozoic basalts from the same region and mid-ocean 52 ridge basalt favor an upper mantle origin (Chen et al., 2007). Further insight was 53 offered by seismic tomography where a horizontally deflected and stagnant 54 Pacific plate at the base of the upper mantle could play a key role in melt 55 generation (Gorbatov and Kennett, 2003; Zhao et al., 2004; Obayashi et al., 2006;

56 Lebedev and van der Hilst, 2008; Li and van der Hilst, 2010). 
Models of seismic velocities are complemented by observations of mantle transition zone (MTZ) discontinuities. For the upper mantle assemblage of olivine composition, phase transitions from olivine to wadsleyite and ringwoodite

60 dissociation are widely accepted origins of the $410 \mathrm{~km}$ discontinuity (from here 61 on, 410) and $660 \mathrm{~km}$ discontinuity (from here on, 660), respectively, at the top 62 and bottom of the MTZ (Anderson, 1967; Ito and Takahashi, 1989). These two 63 mineralogical phase boundaries exhibit opposite Clapeyron slopes (Navrotsky, 64 1980; Ito and Takahashi, 1989; Katsura and Ito, 1989; Weidner and Wang, 1998), 65 and their sensitivity to temperature and composition have been frequently 66 explored in global seismic imaging (Shearer, 1993; Gu et al., 1998; Gu and 67 Dziewonski, 2002; Lawrence and Shearer, 2006a; Deuss, 2009). Based on mantle 68 reflections (Heit et al., 2010; Gu et al., 2012) and conversions ( $\mathrm{Li}$ and Yuan, 69 2003; Liu et al., 2015), a depression in excess of $30 \mathrm{~km}$ has been observed at the 70 base of the MTZ beneath the intraplate volcanic fields in NE China. This 71 topography anomaly coincides with a distinctive low velocity asthenosphere, 72 which has been interpreted as the potential source of melting beneath the volcanic 73 centers in NE China (Zhao et al., 2004; Lei and Zhao, 2005; Niu, 2005; Li and 74 van der Hilst, 2010; Tang et al., 2014).

75 A known source of error in the independent analyses of seismic velocity and 76 discontinuity topography is the trade-off between them (Flanagan and Shearer, 77 1998; Gu and Dziewonski, 2002; Obayashi et al., 2006; Li et al., 2008; Zhao et 
78 al., 2004; Li and van der Hilst, 2010). Time corrections are typically adopted to

79 minimize the excess topography caused by heterogeneous mantle structures,

80 whereas models of seismic velocities are mostly derived under the assumption of

81 unperturbed mantle interfacial depths. This trade-off was reduced by Gu et al.

82 (2003) and Lawrence and Shearer (2006a) through joint inversions of seismic

83 velocity and discontinuity topography, though much of the information embedded

84 in the waveforms of the secondary reflections were underutilized. In this study,

85 we characterize the upper mantle and MTZ beneath the northwestern Pacific

86 region (Figure 1) using waveform inversions of stacked SS precursors (Figure 1d).

87 Our full waveform nonlinear inversion approach enables a simultaneous solution

88 for the travel times of $S S$ precursors, which are sensitive to mantle temperatures

89 surrounding olivine phase boundaries (Ohtani et al., 2004; Deuss, 2009; Lessing

90 et al., 2014), and the impedance contrasts imprinted onto the SS precursors

91 amplitudes (Shearer, 1991; Chambers et al., 2005; Gu and Sacchi, 2009; Lessing

92 et al., 2015). We will demonstrate that a dense precursor dataset alone is sufficient

93 to resolve major upper mantle seismic anomalies in the northwestern Pacific

94 subduction system.

95

96 
99 We utilize a global dataset of broadband and long-period seismograms, 100 recorded between 2006 and 2014, from the Incorporated Research Institutions for 101 Seismology (IRIS). The midpoints of the source-receiver pairs densely sample the 102 structure beneath NE China and the northwestern Pacific subduction zones. We 103 restricted the maximum depth of earthquakes to $75 \mathrm{~km}$ to mitigate the 104 interference between SS precursors and their depth phases (Schmerr and Garnero, 105 2006; An et al., 2007), and adopt a minimum magnitude (Mw) cutoff of 5.5 to 106 ensure sufficient reflection amplitudes. We further constrain the distance from $107100^{\circ}$ to $160^{\circ}$ to minimize the interferences from $\operatorname{ScSSCS}$ (Shearer, 1993; Schmerr 108 and Garnero, 2006) and topside reflections from upper mantle discontinuities. 109 After deconvolving the instrument responses, we apply a Butterworth bandpass 110 filter with corner periods at $15 \mathrm{~s}$ and $75 \mathrm{~s}$ to the transverse component 111 seismograms. We eliminate all traces with signal-to-noise ratio (SNR) less than 1124.0 according to the definition of $\mathrm{Gu}$ et al. (2012), which is more restrictive than 113 the majority of earlier studies due to a substantially larger data volume. The 114 filtered seismograms are then inspected visually to eliminate duplicate records or 115 overlapping events (Schmerr and Garnero, 2006), and a polarity reversal is 116 performed if necessary. This refined dataset contains 7868 waveforms from 1301 117 events. The densities of earthquakes and stations are particularly high in the 118 southern and northern hemispheres, respectively (see Figure 1a). 
119 The amplitudes of SS precursors, which are comparable to those of noise, are 120 greatly enhanced through stacking after aligning the waveforms on the first major 121 swing of $S S$ and normalizing each seismogram with respect to its amplitude. We 122 then deconvolved SS from the transverse component seismograms to minimize 123 source effects. To correct for the move-out, a constant time shift is applied to 124 each waveform based on the differential time between the predicted SS-S410S 125 (Dziewonski and Anderson, 1981) and the reference time for a source-receiver 126 distance of $130^{\circ}$ (Gu and Sacchi, 2009). The move-out corrected seismograms are 127 then time shifted to account for variations in crustal thickness and surface 128 topography using CRUST1.0 (Laske et al., 2013) and ETOPO1 (Amante and 129 Eakins, 2009), respectively. Finally, the time-shifted seismograms are sorted into 130 common midpoint (CMP) circular bins (Shearer, 1991; Gu et al., 2012) along 131 three parallel great-circle transects (profiles A to C) with $3^{\circ}$ inline spacing (see 132 Figure $1 \mathrm{~b}$ ). Circular bins with a radius of $2^{\circ}$ (approximately $15 \%$ overlap between 133 two adjacent bins in the inline direction) are adopted to prevent over-smoothing 134 while providing a sufficient number of traces in each bin for noise suppression. 135 The stacked seismogram in each cap is calculated as the weighted sum of time136 corrected seismograms (Gu and Sacchi, 2009). It is worth nothing that the 137 majority of the source and receiver locations do not overlap with topographic 138 anomalies, e.g. the Rocky Mountains, Alps and Apennines, that may cause 139 confounding observations within the MTZ (Figure 1c). Moreover, while the 
140 azimuthal distribution is dominated by two perpendicular orientations (NE, NW),

141 the crossing ray segments effectively average out the path heterogeneity and 142 enable a reasonable recovery of the velocity and discontinuity depth in most bins

143 (Zheng and Romanowicz, 2012).

146 Figure 1: (a) Global distribution of the earthquake (red stars) and station (blue triangles) locations 147 used in this study. (b) Distribution of the SS bouncepoints from the earthquake-station pairs. The 148 convergent plate boundaries and slab contours are indicated by the red and blue lines, 149 respectively. The contour lines are taken at a constant interval of $50 \mathrm{~km}$ starting at $100 \mathrm{~km}$ depth 150 (Hayes et al., 2012). The SS precursor waveforms are stacked into 30 bins along three parallel 151 profiles A, B and C. (c) Path coverage of the SS precursors that are used in the stacking and 152 inversion procedures. (d) A graphical representation of theoretical ray paths of $S S$ and its 153 precursors for a source-receiver distance of 130.7 degrees. The left panel shows an observed 154 seismogram aligned on the maximum amplitude of the SS phase. S410S and S660 are marked based on the predicted arrival times of PREM (Dziewonski and Anderson, 1981). 
160 A potential pitfall during an $S S$ precursor analysis is the velocity-discontinuity

161 depth trade-off, as the timing of the secondary reflections is corrected based on 162 relatively smooth shear velocity models obtained from earlier studies of body 163 and/or surface waves. An improvement was proposed by jointly inverting for 164 velocity and discontinuity depth using travel times (Gu et al., 2003) and multiple 165 waveforms (Lawrence and Shearer, 2006a). In this study, we quantitatively 166 investigate the waveform information of an $S S$ precursor dataset sampling the 167 northwest Pacific region. We model the full waveforms of SS precursors using the 168 Genetic Algorithm (GA), an effective nonlinear inversion technique (Stoffa and 169 Sen, 1991; Haupt and Haupt, 2004). A similar waveform inversion approach was 170 recently utilized to model the waveforms of P-to-S converted phases (Chen et al., 171 2015), though the emphasis of that study is on the continental crust rather than the 172 MTZ (this study).

173 As a proof of concept we first apply nonlinear inversions to a synthetic 174 seismogram computed using GEMINI 2.2 (Friederich and Dalkolmo, 1995). The 175 Green's function calculation assumes a spherically symmetric Earth model and 176 uses a frequency domain method to numerically solve a system of ordinary first177 order differential equations (Friederich and Dalkolmo, 1995; Friederich, 1999; 178 Fichtner and Igel, 2008). We then utilize GA, a global optimization approach 179 simulating the process of natural evolution (Haupt and Haupt, 2004), to explore 180 solutions of mantle velocity and discontinuity depths that match the simulated 
181 186

$$
J=\left\|W\left(d_{o b s}-d(I \widehat{m})\right)\right\|_{2}^{2}+\mu\|D I \widehat{m}\|_{2}^{2}
$$
al., 2006; Li and van der Hilst, 2010; Ritsema et al., 2011). Due to the

waveform with $10 \%$ noise. We apply a binary-coded genetic algorithm within which variables are defined as indices to a regular discrete search space. Every model parameter (discontinuity depth and velocity perturbations) is represented by a binary string, while the inversion attempts to minimize the following cost function by adjusting the model parameters:

where $I$ is the interpolation operator sampling the model $\widehat{m}$, consisting of shear wave velocities at discontinuities, at a constant depth interval and $D$ is a discrete approximation to the first-order derivative. The symbols $d_{o b s}$ and $d(I \hat{m})$ represent the observed and synthetic seismograms, respectively, and $W$ is the weighting matrix. Finally, $\mu$ is a regularization parameter controlling the trade-off between the first and second terms (i.e., misfit and model norm, respectively), and is determined through trial and error. Our model solution consists of 6 layers, ranging from $220 \mathrm{~km}$ to $720 \mathrm{~km}$ along the depth axis, and the average spacing is $83.3 \mathrm{~km}$. The $S$ velocity is allowed to vary by $\pm 4 \%$ from PREM, which is larger than the frequently reported range below the lithosphere - the primary target of this study (Grand et al., 1997; Mégnin and Romanowicz, 2000; Fukao et al., 2001; Gu and Dziewonski, 2002; Gu et al., 2003; Zhao et al., 2004; Obayashi et interference of the crustal phases and the side-lobes of the main $S S$ arrival, we 
201 mainly target the waveforms corresponding to the structure below $200 \mathrm{~km}$ depth.

202 Our experiments suggest that an average of $1.5 \%$ shear velocity perturbation in 203 the shallow mantle (above $220 \mathrm{~km}$ ), which is comparable to the reported value 204 beneath Northeast China (see Simmons et al. (2010) and Tang et al. (2014)), can 205 cause a $2 \mathrm{~km}$ movement on the MTZ discontinuity depth and a perturbation of $2060.01 \mathrm{~km} / \mathrm{s}$ (or less) on the MTZ velocity. Neither artifacts are significant enough 207 to alter the main observations of this study.

208 We fix the ratio between density and shear velocity based on PREM, and 209 perturbations in density are continuously updated as shear velocities vary during 210 the inversions; a subjective choice that scales between the velocity and density is 211 often adopted to reduce computational cost (Marone et al., 2007; Yuan and 212 Romanowicz, 2010; Liu and Gu, 2012). In general, the arrival times are highly 213 sensitive to velocity and discontinuity depth, whereas the amplitude information 214 embedded in the waveform offers constraints both on velocity and density. While 215 density plays a minor role due to the strong dependence of the objective function 216 on the phase/timing information, a reasonable input density model (PREM in this 217 study) helps stabilize the solutions, especially the shear velocities.

218 Figure 2 shows the results of the nonlinear inversion based on synthetic data 219 with $10 \%$ Gaussian noise. Our input model is modified from PREM, which 220 consists of a low velocity layer atop the 410 and a high velocity layer at the base 221 of the MTZ. We allowed 120 solutions in each generation to ensure diversity at a 
222 manageable computation cost and to steer clear of local extrema. The input and 223 recovered models (see Figure $2 b$ ) exhibit only minimal deviations, which are 224 mainly associated with discretized, relatively coarse, model space. The model evolution history suggests almost immediate convergence to the final model from 226 an initial suite of random models (Figure 2d).

[Figure 2 about here.]

Figure 2: (a) An input transverse component seismogram with 10\% noise (gray) and the inverted 230 trace after 15 generations (black). The precursors are magnified by a factor of 3 and the difference 231 (data-synthetic) seismogram has been offset by -0.08 for clarity. (b) The input (gray) and the 232 recovered (black) model velocities. The red lines indicate the lower and upper boundaries of the model space. (c) The best and mean values of the objective function in each generation. (d) Colorcoded velocity output from nonlinear inversion of the noise-added synthetic seismogram. Random input velocity structures are assumed at the first generation.

\section{Results}


243 the northwestern Pacific region. To evaluate standard errors and the robustness of

244 the observed precursors, we adopt a bootstrapping resampling algorithm (Efron 245 and Tibshirani, 1991; Shearer, 1993; Sacchi, 1998; Deuss, 2009) that takes

246 automatic measurements from 200 randomly selected subsets of the data. During 247 each trial, the amplitudes of the $S 410 S$ and $S 660 S$ are directly determined from 248 the positive peaks associated with the precursors. The amplitude uncertainties of 249 the secondary arrivals are subsequently estimated from the standard deviation of 250 bootstrapped measurements (Deuss, 2009). Overall, the maximum standard error 251 in amplitude is $0.2 \%$ based on bootstrap resampling.

252 Interpolated reflectivity maps are constructed from the $S 410 S$ and $S 660 S$ 253 amplitudes (Figures 3a and 3b), which are defined as maximum amplitudes 254 within a $30 \mathrm{~s}$ time window centered at the respective PREM predicted arrival 255 times of the $S 410 S$ and S660S. Large-scale low and high reflectivity zones are 256 detected, both at the top and the bottom of the MTZ. Two distinctive high 257 amplitude zones are observed on the S410S reflectivity map (see Figure 3a). A 258 SW-NE trending high reflectivity zone is present landward from the Wadati259 Benioff zone on top of the transition zone, extending from the North China 260 Craton to the Wudalianchi volcanic center and reaching a maximum value of $261 \sim 7 \%$ (amplitudes are normalized with respect to the SS phase amplitudes) beneath 262 the Changbai hotspot (see Figure 3a). A second reflectivity zone is observed to 263 the oceanic side of the subducting Pacific plate, approximately $500 \mathrm{~km}$ away 
264 from the Japan trench. The centers of these two anomalies are approximately $2651500 \mathrm{~km}$ apart.

266 Strong S660S reflections are observed in the vicinity of the Wadati-Benioff 267 zone (see Figure 3b). The largest $S 660 S$ amplitude, which attains $\sim 8 \%$ of that of 268 SS, is identified within the reported slab contours (Hayes et al., 2012). While 269 some of the amplitude variations may be affected by the data uncertainties 270 (Figures $3 \mathrm{c}$ and 3d), especially in the southern part of the study region due to low 271 data density, the largest amplitudes of the $S 410 S$ and $S 660 S$ are resolved to $95 \%$ 272 confidence levels.

273 In comparison with PREM, which suggests respective reflection amplitudes of $2743.9 \%$ and $6.1 \%$ relative to $S S$ at a source-receiver distance of $130^{\circ}$ for $S 400 S$ and 275 S670S, the observed values are 1.6-2.3 times higher beneath China (the former) 276 and the Japan Basin (the latter). Part of the amplitude may be affected by the 277 differential attenuation (Q factor) between $S S$ and its precursors. However, our 278 experiment based on conservative choices of Q values suggests a contribution no 279 greater than $0.15 \%$, well within the measurement uncertainties (see 280 supplementary materials). 
Figure 3: The interpolated reflectivity maps of $S 410 S$ (a) and $S 660 S$ (b) amplitude variations. (c) and (d) show the standard errors of the reflection amplitudes based on a bootstrapping analysis. The solid circles denote the centers of the averaging bins and the magenta lines show the slab contours (Hayes et al., 2012). The numbers at the center locations of the averaging bins in panel (d) show the total number of waveforms in each cap used in stacking.

\subsection{Shear Velocity Variation}

We perform nonlinear waveform inversions to simultaneously recover shear wave velocities and the depths of the upper mantle discontinuities. Following the steps of iterative forward modeling procedure described in Section 2.1, the SS precursor waveforms are inverted based on 15 generations, and 120 simulated waveforms are generated in each generation through parallel programming. For consistency, the synthetic waveforms are filtered between the same corner 298 periods as the observed data and the source mechanism is removed by isolating 299 and deconvolving SS (Figure 4). In general, the waveforms of S410S and S660S 300 are accurately modeled by the synthetic seismograms, especially in view that 1)

301 PREM contains no velocity and density jumps at mid MTZ depths (Dziewonski 302 and Anderson, 1981), causing slight mismatches of the waveforms between 
S410S and S660S, and 2) the expected misfit between the data and synthetics is nonzero due to the uncertainties (e.g., random noise and imperfect structural corrections) in precursor amplitude and arrival time. The $520 \mathrm{~km}$ discontinuity

306 (for short, the 520) is locally enhanced within the Wadati-Benioff zone (Gu et al., 307 2012), though its lateral variations are complex in all three cross-sections (see 308 Figure 4).

[Figure 4 about here.]

311 Figure 4: SS precursor waveform inversion results for profiles A to C. In all panels the black and 312 red lines represent the observed and synthetic stacks, respectively. The key features in the 313 waveform data are highlighted using the dashed boxes. The symbols D and E represent areas with 314 depressed and elevated discontinuity topography, respectively. The letters $\mathrm{H}$ and $\mathrm{L}$ indicate the 315 high and low amplitude precursory arrivals, respectively.

We determine a local 1D velocity model for each cap based on the stacked $S S$ 318 precursor waveform. A 3D model of the study region is then constructed from the 319 resultant 1D models using a linear interpolation algorithm. The inverted shear 320 velocities under NE China and the northwestern Pacific subduction regions 321 (Figure 5) highlight the morphology of the Japan-Kuril trench, showing an 322 average velocity in excess of $1.5 \%$ relative to PREM. This high velocity structure 323 intersects the base of the upper mantle near the Sea of Japan and extends 
324 westward toward Central Asia. Its presence and the contrasting discontinuity 325 topography of the 410 (elevated by $10-25 \mathrm{~km}$ ) and 660 (depressed by $10-27 \mathrm{~km}$ ) 326 are required to match the low amplitude precursory arrivals (see Figures 3 and 4). 327 The presence of a high velocity anomaly (cold subducting oceanic lithosphere) 328 within the MTZ can reduce the impedance contrast across the $660 \mathrm{~km}$ boundary 329 and produce low S660S amplitudes (see Figures 4 and 5). The eastern side of this 330 dipping high velocity structure shows a distinct low velocity zone, reaching its 331 minimum value (approximately $1.5 \%$ slower than that of PREM) within the MTZ 332 beneath the northeastern Honshu arc. The presence of this anomaly is required by 333 a high amplitude, early-arriving $S 410 S$ oceanward of the subducting Pacific plate 334 (see Figures 3 and 4), which is partially accommodated by a relative depression $335(\sim 15 \mathrm{~km})$ of the 410 . This low velocity structure appears to continue to the base 336 of the MTZ under the Japan and Ryukyu trenches.

337 The most intriguing low velocity zone is observed in the upper mantle beneath 338 NE China, potentially extending $1000 \mathrm{~km}$ further inland from the eastern end of 339 the Wudalianchi volcanic belt to the northwestern facade of the Greater Hinggan 340 Mountains (see Figures 5a and 5b). The center, and the deepest part, of this broad 341 anomaly resides beneath the Changbai Mountain Range, initiating at shallow 342 depths and persisting down to a depth of $\sim 500 \mathrm{~km}$ (see Figures $5 \mathrm{a}-\mathrm{c}$ ). This low 343 velocity structure is accompanied by a $5-10 \mathrm{~km}$ depression of the 410 , which 344 reflects an increased SS-S410S differential time in this region (see Figure 4). The 
345 observed low velocity structure under the Wudalianchi volcanic field is limited to

346 depths above $350 \mathrm{~km}$, which is significantly shallower in comparison with that 347 beneath the Changbai hotspot.

350 Figure 5: Lateral variations in $S$-wave velocity relative to PREM (Dziewonski and Anderson, 1981) at $300 \mathrm{~km} \mathrm{(a),} 400 \mathrm{~km}(\mathrm{~b}), 500 \mathrm{~km}(\mathrm{c})$, and $600 \mathrm{~km}$ (d) depths beneath NE China and the northwestern Pacific region. The blue and red colors represent fast and slow anomalies, respectively. The solid circles mark the centers of the averaging caps and the white triangles

354 denote the locations of major intraplate volcanoes.

355

Changes in MTZ thickness across the study region, which are calculated from the inverted transition zone discontinuities depths (Figure 6a), are consistent with those of Gu and Dziewonski (2002) using a correlation based method (Figure 6b). Both studies show increased MTZ thickness north of the Ryukyu trench and 360 beneath the Songliao Basin in northeastern China, especially in the present study 361 where values exceed the global average of $242 \mathrm{~km}$ ( $\mathrm{Gu}$ et al., 1998; $\mathrm{Gu}$ and Dziewonski, 2002; Lawrence and Shearer, 2006b). The largest difference, which reflects improvements in methodology and vastly improved spatial resolution, is 364 an anomalously thick MTZ in this study beneath Northeast China due to a 25-30 $365 \mathrm{~km}$ depression of the 660 (see Z1 in Figure 6a). This anomaly is consistent with 366 the recent findings of Liu et al. (2015) based on migrated receiver functions (see 
367 the dashed-line box, Figure 6a). To a lesser extent, the MTZ beneath the Ryukyu

368 trench is approximately $15 \mathrm{~km}$ thicker in this study than $\mathrm{Gu}$ and Dziewonski 369 (2002), despite similar shapes and signs (see Z2 in Figure 6a).

370 As will be discussed in Section 4, the combination of a depressed and amplified 371410 , a severely deformed 660 and a low velocity asthenosphere beneath the 372 Changbai-Wudalianchi hotspot has major implications for the intraplate 373 volcanism in NE China.

376 Figure 6: (a) MTZ thickness variations inferred from the inverted depths of the 410 and 660 by 377 this study. (b) MTZ thickness variations reported by Gu and Dziewonski (2002). The red and blue 378 colors represent regions of narrow and thick MTZ, respectively. Z1 and Z2 indicate anomalously 379 thick MTZ beneath NE China and the Ryukyu trench, respectively. The dashed-line box in panel 380 (a) outlines a relatively thick MTZ (>270 km) in Liu et al. (2015), obtained from depth migrated 381 P-to-S converted waves.

\section{Discussion}

\subsection{General Assessment}


This study presents a nonlinear optimization method that accurately determines mantle large-scale shear velocity and discontinuity topography based on SS waveform information alone. In comparison with a recent study of SS precursors 390 for the same region (Gu et al., 2012), the current study benefits from denser data 391 coverage, especially in the northern flank of the study region where data density 392 increased by $\sim 50 \%$ due to four extra years of recordings. The resolution improved 393 further from the SS precursors amplitude information, which is essential for 394 resolving the impedance contrast at major seismic discontinuities, as well as from 395 the reduced trade-off between the velocity and discontinuity depth. While the 396 scaling of the reported features $(>300 \mathrm{~km})$ may be smaller than the nominal 397 resolution $(>1200 \mathrm{~km}$ ), the use of multiple stations (Rost and Thomas, 2009; 398 Schmerr and Thomas, 2011) and dense sampling (Cao et al., 2011; Gu et al., 399 2012; Zheng and Romanowicz, 2012) can significantly reduce the effective 400 Fresnel zone and smearing along an isochron (Rost and Thomas, 2009; Cao et al., $4012010)$.

402 The westward dipping high velocity structure beneath the back-arc region 403 (Figures 7a-c), which is consistent with earlier findings from $P$ and $S$-wave travel 404 times (van der Hilst et al., 1991; Fukao et al., 1992; Gorbatov and Kennett, 2003; 405 Huang and Zhao, 2006; Obayashi et al., 2006; Li and van der Hilst, 2010; Zhao et 406 al., 2012) and waveform (Mégnin and Romanowicz, 2000; Friederich, 2003) 407 inversions, has been widely associated with subducted Pacific lithosphere beneath 
408 Japan and Kuril islands. The general morphologies of the high velocity structures 409 are concordant with those of Obayashi et al. (2006) (see Figures 7d-f), both of 410 which are well supported by the locations of deep focus earthquakes in the study 411 region.

412 The fate of the subducted Pacific plate has been a source of considerable 413 debates (van der Hilst et al., 1993; Grand, 2002; Huang and Zhao, 2006; Schmerr 414 and Thomas, 2011). While relatively unimpeded penetration into the lower 415 mantle has been documented along Mariana (Creager and Jordan, 1986; Bijwaard 416 et al., 1998; Zhao et al., 2004; Huang and Zhao, 2006; Li et al., 2008) and Kuril 417 (Jordan, 1977; Li et al., 2008; Schmerr and Thomas, 2011; Gu et al., 2012) 418 islands, substantial deformation and changes in slab dip have been frequently 419 documented at the base of the upper mantle as evidence of slab deflection or 420 bending (Zhao et al., 2004; Fukao et al., 2001; Huang and Zhao, 2006; Gu et al., 421 2012). Beneath the Sea of Japan, the seismic structure in the transition zone is 422 dominated by a $1.5-2.0 \%$ increase in $P$ velocity (Fukao et al., 1992; Huang and 423 Zhao, 2006; Obayashi et al., 2006), but the deflected slab segment is less well 424 imaged by shear velocities across the study region (Fukao et al., 2009; Gorbatov 425 and Kennett, 2003). The result of our waveform inversion of SS precursor 426 provides further evidence for slab deflection below the Sea of Japan and 427 stagnation beneath eastern China (see Figures 7a-c). 
430 Figure 7: (a)-(c) West-east vertical cross-sections of the inverted shear wave velocity anomalies 431 along profiles A to $\mathrm{C}$ from 200 to $720 \mathrm{~km}$ depth beneath the northwestern Pacific subduction 432 zones and NE China. The magenta circles indicate the locations of deep earthquakes within a $2^{\circ}$ 433 distance from each profile. The depths of the 410 and $660 \mathrm{~km}$ discontinuities are indicated by the 434 dashed lines. A1 and A2 mark the downgoing and stagnant parts of the Pacific slab, respectively, 435 while A3 and A4 denote low shear velocity anomalies beneath the Changbai-Wudalianchi hotspot. 436 A5 indicates low seismic velocities on the ocean side of the Wadati-Benioff zone. (d)-(f) Similar 437 to (a)-(c), but for an earlier model of $P$-wave velocities (Obayashi et al., 2006).

The simultaneous solutions of velocity and discontinuity depth enable a detailed examination of the effective mantle temperatures within the MTZ (Schmerr and 444 Garnero, 2006). On the global scale, the depths of the 410 and 660 are either 445 uncorrelated (Flanagan and Shearer, 1998; Gu et al., 1998) or slightly 446 anticorrelated (Revenaugh and Jordan, 1991; Gossler and Kind, 1996; Li et al., 447 2003; Gu et al., 2003). The lack of correlation is largely attributable to smaller448 scale heterogeneities (Schmandt and Humphreys, 2010), compositional variations 449 (Weidner and Wang, 1998; Schmerr and Garnero, 2007), or non-vertical 
450 structural geometry (Gu et al., 2012). Considerations for localized dipping 451 thermal-chemical structures are crucial in these correlation analyses, especially in 452 the vicinity of major subduction zones. To properly account for slab dip, we 453 compute the correlation coefficients between MTZ thickness and the average 454 MTZ shear velocity for slab dip angles ranging from 15 to 90 degrees. The 455 maximum correlation coefficient (0.72) from all three profiles is determined at 30 456 degrees, which is $22 \%$ higher than the uncorrected (90 degrees dip) value (Figure 457 8). This effective slab dip is in excellent agreement with previously reported 458 values (van der Hilst et al., 1993; Gudmundsson and Sambridge, 1998; Huang 459 and Zhao, 2006; Hayes et al., 2012), which highlights 1) the need to properly 460 consider inclined slab morphology, and 2) the dominance of thermal, rather than 461 compositional, variations within the MTZ. A closer examination of the 462 correlation coefficient at the optimal slab dip (see Figures 8c and 8d) suggests 463 substantial improvements over the conventional vertical structure assumption in 464 global analyses (Flanagan and Shearer, 1998; Gu et al., 1998; Tauzin et al., 2008; 465 Houser and Williams, 2010). The strength of the correlation (0.72) largely reflects 466 the severity of thermal perturbations, relative to the ambient mantle, within the 467 subducting oceanic lithosphere. It is enhanced further by a dipping low velocity 468 structure east of the Wadati-Benioff zone (see A5 in Figures $7 \mathrm{a}$ and $7 \mathrm{~b}$ ), which 469 has been previously reported by Obayashi et al. (2006) and Gu et al. (2012) in 470 possible connection with 1) convective return flow in connection with slab 
471 dynamics (Bercovici and Karato, 2003; Ohtani et al., 2004), and 2) partial 472 subduction of a residual Mesozoic hot thermal plume (Larson, 1991; Tatsumi et 473 al., 1998; Honda et al., 2007).

[Figure 8 about here.]

476 Figure 8: (a) A cross-section of our shear velocity model along profile C. The dashed and solid 477 straight lines indicate how the MTZ thickness and average MTZ velocity are measured along slab 478 dips of 30 and 45 degrees, respectively. (b) The correlation between MTZ thickness and the 479 average MTZ shear velocity perturbations at a range of dip angles. (c) Observations of apparent 480 MTZ thickness versus the apparent average MTZ shear velocity along slab dip of 30 degrees. The 481 least-square fit and correlation coefficient are also shown. (d) Similar to (c) but for slab dip of 90 482 degrees.

A notable seismic anomaly that reduces the anticorrelation between the depths of 410 and 660 is the low velocity zone beneath the Changbai-Wudalianchi

489 hotspot. The geometry of this low velocity structure (see A3 and A4 in Figures 7b 490 and 7c) is in excellent agreement with those from earlier studies of this proposed 491 back-arc region (Tatsumi et al., 1990; Lei and Zhao, 2005; Huang and Zhao, 
492 2006; Li and van der Hilst, 2010; Tang et al., 2014). This anomaly has been

493 linked to the horizontally deflected Pacific slab and the subsequent upwelling of 494 hot asthenospheric material (Tatsumi et al., 1990; Li and van der Hilst, 2010; 495 Zhao and Liu, 2010), though more recent studies (Tang et al., 2014; Liu et al., 496 2015) have suggested a slab gap west of the stagnant slab.

497 In this study, depressed 410 and 660 beneath the Changbai and Wudalianchi 498 volcanic fields imply discontinuous seismic velocities within the MTZ (see A2 in 499 Figures $7 \mathrm{~b}$ and $7 \mathrm{c}$ ), above which a low velocity structure (see A3 and A4 in 500 Figures $7 \mathrm{~b}$ and $7 \mathrm{c}$ ) continues to the shallow mantle (Figure 11). Anomaly A3 501 appears to terminate at mid-MTZ depths $(\sim 500 \mathrm{~km})$, which is moderately deeper 502 than earlier estimates (Zhao and Ohtani, 2009; Li and van der Hilst, 2010). 503 Judging from the MTZ velocity characteristics, the transition from low to high 504 velocity appears to be gradational rather than abrupt. The presence of the low 505 velocity zone does not significantly impact the amplitude of the 520, which is 506 diffuse above the stagnant part of the Pacific slab (see Figures $4 b$ and $7 b$ ).

507 To examine the robustness and resolution of the velocity and discontinuity 508 depth solutions we conduct a hypothesis test, a frequently adopted technique for 509 linearized inversions. In this test, we first introduce a low velocity structure 510 resembling that beneath the Changbai hotspot (Figure 9). This hypothetical 511 anomaly terminates near $410 \mathrm{~km}$, overlying a high velocity structure similar to 512 the stagnant oceanic lithosphere. The input depths of the 410 and $660 \mathrm{~km}$ 
513 discontinuities are both depressed by more than $10 \mathrm{~km}$ near the largest shear

514 velocity perturbations. After the synthetic seismograms are computed for each 515 cap location based on these input parameters, we add up to $0.2 \%$ Gaussian noise 516 depending on the observed standard errors from bootstrap resampling (Section

517 3.1). The recovered models through the same inversion procedure show two well518 defined seismic velocity anomalies with the same signs and shapes as the input 519 (see Figure 9). The maximum amplitudes of the recovered anomalies are about $52080 \%$ and $95 \%$ of the assumed values of the respective low and high velocities; the 521 recovery of the latter anomaly is superior due to a larger, more laterally coherent 522 input structure. The amplitudes near the edges of the major anomalies are 523 preferentially reduced due to the lateral averaging with a substantially weaker 524 structure to the west. The depressions of the phase boundaries near the center of 525 the profile are recovered to about $90-95 \%$ relative to the input values. The 526 performance of the inversion is equally effective at recovering a relatively 527 shallow low velocity zone beneath the Wudalianchi hotspot. In this case, the 528 inversion recovers over $75 \%$ of the maximum input velocity and discontinuity 529 topography, as well as a minor lateral gradient east of the volcanic center (see 530 Figure 9). Our hypothesis test demonstrates that 1) a restoring resolution analysis 531 is equally effective in nonlinear inversions as their linear counterpart, and 2) the 532 morphologies of the low velocity zones beneath the volcanic centers and the 533 underlying stagnant slab can be sufficiently resolved in our study region. 
[Figure 9 about here.]

536 Figure 9: Input models (panels a and b) and the inverted results (panels c and d) of the synthetic

537 tests along profiles B and C. The depths of the 410 and 660 are indicated by the thick dashed 538 lines.

539

540 To further validate the recovered images from the $1 \mathrm{D}$ waveform inversions of

541 SS precursors (see Figure 7), we implement a 2D waveform tomography using the 542 same nonlinear inversion technique (Figure 10). The mantle is discretized into 543 cells of $3^{\circ}$ (in the horizontal direction) by $50 \mathrm{~km}$ (in the vertical direction). To 544 simplify the problem, we assume known discontinuity depths from 1D inversions 545 (see Figure 7) and solve for perturbations of shear velocities within each cell; it is 546 worth noting that we define a finer vertical cell size of $10 \mathrm{~km}$ around the 547 discontinuity depths (220, 300, 400, 520 and $670 \mathrm{~km}$ depths). In this approach, 548 the travel times of the precursors and their corresponding reflection coefficients 549 are calculated using the 2D ray tracing method (Lebedev and Nolet, 2003; Zhao 550 et al., 2004; Lawrence and Shearer, 2006a; Priestley et al., 2006). Then, the 551 reflectivity profiles are convolved with the source time functions extracted from 552 the stacked waveforms (Shearer et al., 1999). We generate over 2000 solutions at 553 each generation, while $5 \%$ of the initial random solutions are replaced by shear 554 velocities from GyPSuM (Simmons et al., 2010). The optimized synthetic 555 waveforms from 2D nonlinear inversions closely match the observations along 
profile B (see Figure 10b). The velocity model shows a westward dipping

557 structure similar to that recovered by 1D waveform inversions, which appears to 558 flatten toward NE China. The maximum velocity of the slab (1.5\%) is comparable 559 to that of $1 \mathrm{D}$ inversion, whereas the low velocity zone above the $410(-1.1 \%)$ is 560 slightly weaker than the 1D counterpart. Part of the amplitude difference of the 561 low velocity zone between 1D and 2D inversion outcomes could be explained by the inclusion of a more realistic, low velocity lithosphere in the latter experiment.

Figure 10: 2D waveform tomography results using the stacked waveforms observed along profile B (see Figure 4). The results clearly show a high velocity structure, associated with the subducting 567 plate, and reduced shear wave velocities beneath NE China. (a) Schematic view of the SS ray 568 paths used in waveform tomography. The sources and receivers are indicated by stars and 569 triangles, respectively. The thick black lines outline the area covered by the waveform 570 tomography approach. The outlined area is zoomed on from $200 \mathrm{~km}$ to $750 \mathrm{~km}$ depth. (b) The 571 visual comparison between the observed (black) and synthetic (red) waveforms shows a good 572 agreement between the input and modeled waveforms. (c). The normalized fitness values of the 573 objective function in each generation. (d) Color-coded cross-section (outlined area in panel a) of 574 the shear wave velocity perturbations. The blue and red colors represent the fast and slow 575 velocities, respectively. The key features of the recovered image are also labeled (LVZ stands for 576 low velocity zone). 
578 Our models consistently suggest a relatively warm mantle in the vicinity of the $579410 \mathrm{~km}$ discontinuity. The observed $5-10 \mathrm{~km}$ depression of the $410 \mathrm{~km}$ seismic 580 discontinuity beneath the intraplate volcanic fields in mainland China can be 581 attributed to the response of the olivine to wadsleyite phase transition, an 582 exothermic reaction with a positive Clapeyron slope of 1.5-4.0 $\mathrm{MPaK}^{-1}$ (Akaogi 583 et al., 1989; Houser and Williams, 2010; Lessing et al., 2014), to the presence of 584 a high temperature anomaly above or across this boundary. By assuming a 585 temperature dependence of the shear wave velocity $\left(\frac{\partial \ln V_{S}}{\partial T}\right)$ of $-7 \times 10^{-5} / \mathrm{K}$ for a dry 586 upper mantle (Houser and Williams, 2010), a $S$-wave velocity reduction of $1.5 \%$ 587 results in an approximate temperature increase of $215 \mathrm{~K}$ relative to the average 588 value of $1694 \mathrm{~K}$ for the olivine phase change (Houser and Williams, 2010). The 589 phase boundary moves toward the higher pressures (by 320-860 MPa) and greater 590 depths, which are consistent with recent reports of 20-30 km of depression in the 591 same region (Li et al., 2000; Li and Yuan, 2003; Liu et al., 2015). Different 592 mechanisms have been proposed to explain the origin of widely distributed low 593 velocity regime in the upper mantle beneath northeastern China, most of which 594 are associated with the subducting Pacific plate. An incomplete list of these 595 mechanisms include 1) partial melting induced by the deep dehydration of the 596 slab (Zhao and Ohtani, 2009), 2) passive upwelling of asthenospheric materials in 597 response to slab accumulation within the transition zone (Bercovici and Karato, 
598

599

600

601

602

603 elevate the olivine to wadsleyite phase boundary. While the extent of the 604 elevation in presence of water remains questionable (Frost and Dolejš, 2007;

605 Schmerr and Garnero, 2007), an elevated 410 is simply inconsistent with either 606 the observed depression from this (see Figure 7) and earlier studies (Li and Yuan, 607 2003; Liu et al., 2015), or the reported water content in this region (Ichiki et al., 608 2006; Chen et al., 2007; Fukao et al., 2009; Ye et al., 2011). In a case of water 609 saturated mantle, a hydrous melt lens may form atop the 410 and produce 610 multiple phase transitions. This was reported in South America where a relatively 611 deep, modest wet to dry wadsleyite reflection was detected at $415 \mathrm{~km}$ (Schmerr 612 and Garnero, 2007). Based on numerical experiment from the same study, the 613 expected amplitude (which increases with water content) is significantly lower 614 than the predicted value from PREM and far below our observations under the 615 Changbai hotspot.

616 A favorable solution for the observed 410 amplitude and depth will require 617 significantly increased impedance contrast across a depressed 410, which could 618 be satisfied by the presence of a melt layer in a relatively dry, warm mantle. 
619 Increasing $\mathrm{Mg}$ by up to $4 \%$ within the melt layer can effectively depress the 410 $620 \mathrm{~km}$ discontinuity by about $10 \mathrm{~km}$ (Fei and Bertka, 1999; Schmerr and Garnero, 621 2007), which corroborates our observations in NE China. Both decompression 622 melting associated with passive upwelling (Bercovici and Karato, 2003; Faccenna 623 et al., 2010) and a significant sub-slab component from the slab window (Tang et 624 al., 2014) can induce partial melt atop the 410. This would depress the 410 phase 625 boundary, enhance the impedance contrast and substantially increase the $S$-wave 626 reflection amplitude, which are supported by our observations beneath the 627 Changbai hotspot. Insufficient resolution around the suggested slab window (see 628 Figures 7 and 11) makes it difficult to clearly differentiate between these two 629 candidate mechanisms, however.

630 Further inferences could be made regarding the mantle beneath the two volcanic 631 centers examined in this study. Despite the considerable depth difference between 632 the Changbai (about $500 \mathrm{~km}$ ) and Wudalianchi (about $350 \mathrm{~km}$ ) hotspots, trace 633 element analyses (Basu et al., 1991; Kuritani et al., 2009) suggest a comagmatic 634 upper mantle origin with EM1 (Enriched Mantle 1) composition (Jackson and 635 Dasgupta, 2008). Hence, it is convincible that the mantle upwelling beneath the 636 Changbai hotspot is responsible for the source of partial melt for both volcanic 637 centers in view of its greater vertical extent and strength (see Figures 5a and 11). 638 Further work will be needed to quantify the accuracies of the aforementioned 639 mechanisms and interpretations. Finally, increased seismic velocities at the base 
640 of the MTZ under northeast China and a deep $660(>680 \mathrm{~km})$ relative to the 641 regional average of 655-665 km (Shearer, 1993; Gu et al., 2003; Houser and 642 Williams, 2010) are consistent with the respective measurements based on 643 analyses of receiver functions ( $\mathrm{Li}$ et al., 2000; Li and Yuan, 2003; Liu et al., 6442015 ) and migration of long period $S S$ precursors (Gu et al., 2012). A maximum 645 depression of $20-30 \mathrm{~km}$ is reported on the 660 , possibly in association with a 646 decrease of 300-400 K in MTZ temperature (Li and Yuan, 2003; Ye et al., 2011). 647 A negative temperature anomaly of $\sim 300 \mathrm{~K}$ and a greater depth of the $660 \mathrm{~km}$ 648 discontinuity jointly suggest increased $S$-wave velocities of approximately $2 \%$ in 649 the presence of a negative Clapeyron slope from the post-spinel phase transition 650 (Houser and Williams, 2010; Lessing et al., 2014). The low temperature anomaly 651 and locally depressed 660 could be explained by the existence of water within the 652 MTZ, the amount of which depends on the volume entrained within the slab as 653 well as the residence time of the slab (Fukao et al., 2009). $P$ and $S$ waveform 654 modeling from northeast China detects a $130 \mathrm{~km}$ thick high velocity zone at the 655 base of the MTZ, which may contain up to $0.3 \mathrm{wt} \%$ water based on estimates 656 from Ye et al. (2011). 
659 Figure 11: (a) Isosurfaces showing the major seismic anomalies beneath Northeast China and the 660 northwestern Pacific subduction zones in the depth range of 200 to $700 \mathrm{~km}$. The blue and red 661 colors represent fast and slow shear velocities, respectively, at the respective isosurfaces of $662+0.65 \%$ and $-1.0 \%$. The definitions of major seismic anomalies (A1-A5) are the same as in Figure

663 7. The black lines show the slab contours, which are taken at $50 \mathrm{~km}$ depth intervals starting at 100 $664 \mathrm{~km}$ (Hayes et al., 2012), and the magenta circles indicate the locations of deep-focus earthquakes. 665 (b) A schematic illustration of a NW-SE vertical section (see Figure 11a) showing the possible 666 origins of the intraplate volcanism in Northeast China. Three possible mechanisms for the 667 intraplate volcanism (discussed in Section 4.3) are also illustrated: (1) deep dehydration process of 668 the slab, (2) passive upwelling in response to the subduction of the Pacific plate, and (3) escaping 669 of the hot sub-slab material through a slab window.

\section{Conclusions}

Our study demonstrates that shear wave velocity and the topography of MTZ discontinuities beneath northeastern China and the northwestern Pacific region 676 can be simultaneously recovered using the waveforms of $S S$ precursors alone. A 677 statistically significant positive correlation is observed between MTZ thickness 678 and the average MTZ velocity along an effective slab dip of $~ 30$ degrees, which 
679 reflects the dominant effect of thermal heterogeneity in the upper mantle and 680 MTZ. A strong low velocity zone is found beneath active volcanoes in 681 northeastern China, persisting to a depth of $>500 \mathrm{~km}$, which implies that the 682 active volcanoes in eastern China (Changbai and Wudalianchi hotspots) may be 683 fueled by a hot thermal anomaly from MTZ depths.

684 Passive upwelling and/or a slab window could be responsible for a relatively 685 water-poor melt layer atop the 410 , which cause $5-10 \mathrm{~km}$ depression and 686 substantially enhance reflection amplitude on the olivine to wadsleyite transition.

687 A distinctive low velocity structure is also observed east of the Wadati-Benioff 688 zone, which narrows MTZ by $30 \mathrm{~km}$ relative to its regional average. This low 689 velocity zone is possibly associated with (i) a fossil superplume ( 140 Ma) or (ii) 690 upwelling hot mantle material in response to slab and lower mantle interaction. 691 Overall, our findings from nonlinear SS precursor waveform inversions offer a 692 new window into the process and fate of the subducting oceanic plate in the 693 northwestern Pacific region. From a technical stand point, the nonlinear joint 694 inversion technique introduced in this study represents a new phase in the 695 retrieval of seismic properties pertaining to large scale thermal/chemical 696 structures in the mantle. This is not aimed to be a replacement of the existing 697 travel time and waveform tomographic approaches, especially in view of the 698 relatively coarse spatial resolution of the $S S$ precursor dataset, but an 
699 improvement that effectively incorporates the amplitudes of secondary reflections 700 or conversions from mantle interfaces.

701

702

703 Acknowledgment

704

705 We thank Yunfeng Chen, RuijiaWang and Yuanyin Zhang for their assistance 706 during the preparation of this manuscript. We also thank two anonymous 707 reviewers for their insightful suggestions and comments. The facilities of IRIS 708 Data Services, and specifically the IRIS Data Management Center, were used for 709 access to waveforms, related metadata, and/or derived products used in this study. 710 IRIS Data Services are funded through the Seismological Facilities for the 711 Advancement of Geoscience and EarthScope (SAGE) Proposal of the National 712 Science Foundation under Cooperative Agreement EAR-1261681. This study is 713 supported by the Helmholtz-Alberta Initiative (HAI) and National Science and 714 Engineering Council (NSERC). 
Akaogi, M., Ito, E., Navrotsky, A., 1989. Olivine-modified spinel-spinel transitions in the system $\mathrm{Mg}_{2} \mathrm{SiO}_{4}-\mathrm{Fe}_{2} \mathrm{SiO}_{4}$ : calorimetric measurements, thermochemical calculation, and geophysical application. Journal of Geophysical Research 94, 15671-15685.

Amante, C., Eakins, B., 2009. ETOPO1 1 Arc-Minute Global Relief Model:

An, Y., Gu, Y.J., Sacchi, M.D., 2007. Imaging mantle discontinuities using least squares radon transform. Journal of Geophysical Research 112:B10303. doi:10.1029/2007JB005009.

Anderson, D.L., 1967. Phase changes in the upper mantle. Science 157, 11651173.

Basu, A.R., Junwen, W., Wankang, H., Guanghong, X., Tatsumoto, M., 1991. Major element, $\mathrm{REE}$, and $\mathrm{Pb}, \mathrm{Nd}$ and $\mathrm{Sr}$ isotopic geochemistry of Cenozoic volcanic rocks of eastern China: implications for their origin from suboceanic-type mantle reservoirs. Earth and Planetary Science Letters 105, 149-169.

Bercovici, D., Karato, S.i., 2003. Whole-mantle convection and the transitionzone water filter. Nature 425, 39-44.

Bijwaard, H., Spakman, W., Engdahl, E.R., 1998. Closing the gap between regional and global travel time tomography. Journal of Geophysical Research 103, 30055-30078.

Campbell, I.H., 2007. Testing the plume theory. Chemical Geology 241, 153-176. 
Cao, Q., Van der Hilst, R., De Hoop, M., Shim, S.H., 2011. Seismic imaging of transition zone discontinuities suggests hot mantle west of hawaii. Science $332,1068-1071$.

Cao, Q.,Wang, P., Van der Hilst, R., De Hoop, M., Shim, S.H., 2010. Imaging the upper mantle transition zone with a generalized radon transform of SS precursors. Physics of the Earth and Planetary Interiors 180, 80-91.

Chambers, K., Deuss, A., Woodhouse, J., 2005. Reflectivity of the 410-km discontinuity from PP and SS precursors. Journal of Geophysical Research 110:B02301. doi:10.1029/2004JB003345.

Chen, Y., Gu, Y.J., Dokht, R.M., Sacchi, M.D., 2015. Crustal imprints of Precambrian orogenesis in western Laurentia. Journal of Geophysical Research 120, 6993-7012.

Chen, Y., Zhang, Y., Graham, D., Su, S., Deng, J., 2007. Geochemistry of Cenozoic basalts and mantle xenoliths in Northeast China. Lithos 96, 108126.

Creager, K.C., Jordan, T.H., 1986. Slab penetration into the lower mantle beneath the Mariana and other island arcs of the northwest Pacific. Journal of Geophysical Research 91, 3573-3589.

Deuss, A., 2009. Global observations of mantle discontinuities using SS and PP precursors. Surveys in Geophysics 30, 301-326.

Dziewonski, A.M., Anderson, D.L., 1981. Preliminary reference Earth model. Physics of the Earth and Planetary Interiors 25, 297-356.

Efron, B., Tibshirani, R., 1991. Statistical data analysis in the computer age. Science 253, 390-395.

Faccenna, C., Becker, T.W., Lallemand, S., Lagabrielle, Y., Funiciello, F., Piromallo, C., 2010. Subduction-triggered magmatic pulses: A new class of plumes? Earth and Planetary Science Letters 299, 54-68. 
Fei, Y., Bertka, C.M., 1999. Phase transitions in the Earth's mantle and mantle mineralogy. Mantle petrology: field observations and high pressure experimentation $6,189-207$.

Fichtner, A., Igel, H., 2008. Efficient numerical surface wave propagation through the optimization of discrete crustal models - a technique based on non-linear dispersion curve matching (DCM). Geophysical Journal International 173, 519-533.

Flanagan, M.P., Shearer, P.M., 1998. Global mapping of topography on transition zone velocity discontinuities by stacking SS precursors. Journal of Geophysical Research 103, 2673-2692.

Friederich, W., 1999. Propagation of seismic shear and surface waves in a laterally heterogeneous mantle by multiple forward scattering. Geophysical Journal International 136, 180-204.

Friederich, W., 2003. The S-velocity structure of the East Asian mantle from inversion of shear and surface waveforms. Geophysical Journal International $153,88-102$.

Friederich, W., Dalkolmo, J., 1995. Complete synthetic seismograms for a spherically symmetric earth by a numerical computation of the Green's function in the frequency domain. Geophysical Journal International 122, 537-550.

Frost, D.J., Dolejš, D., 2007. Experimental determination of the effect of $\mathrm{H}_{2} \mathrm{O}$ on the 410-km seismic discontinuity. Earth and Planetary Science Letters 256, 182-195.

Fukao, Y., Obayashi, M., Inoue, H., Nenbai, M., 1992. Subducting slabs stagnant in the mantle transition zone. Journal of Geophysical Research 97, 48094822.

Fukao, Y., Obayashi, M., Nakakuki, T., 2009. Stagnant slab: a review. Annual Review of Earth and Planetary Sciences 37, 19-46. 
Fukao, Y., Widiyantoro, S., Obayashi, M., 2001. Stagnant slabs in the upper and lower mantle transition region. Reviews of Geophysics 39, 291-323.

Gorbatov, A., Kennett, B., 2003. Joint bulk-sound and shear tomography for Western Pacific subduction zones. Earth and Planetary Science Letters 210, 527-543.

Gossler, J., Kind, R., 1996. Seismic evidence for very deep roots of continents. Earth and Planetary Science Letters 138, 1-13.

Grand, S.P., 2002. Mantle shear-wave tomography and the fate of subducted slabs. Philosophical Transactions of the Royal Society of London A: Mathematical, Physical and Engineering Sciences 360, 2475-2491.

Grand, S.P., van der Hilst, R.D., Widiyantoro, S., 1997. Global seismic tomography: a snapshot of convection in the earth. GSA Today 7, 1-7.

Gu, Y., Dziewonski, A.M., Agee, C.B., 1998. Global de-correlation of the topography of transition zone discontinuities. Earth and Planetary Science Letters 157, 57-67.

Gu, Y.J., Dziewonski, A.M., 2002. Global variability of transition zone thickness. Journal of Geophysical Research 107:2135. doi:10.1029/2001JB000489.

Gu, Y.J., Dziewonski, A.M., Ekström, G., 2003. Simultaneous inversion for mantle shear velocity and topography of transition zone discontinuities. Geophysical Journal International 154, 559-583.

Gu, Y.J., Okeler, A., Schultz, R., 2012. Tracking slabs beneath northwestern Pacific subduction zones. Earth and Planetary Science Letters 331, 269-280.

Gu, Y.J., Sacchi, M., 2009. Radon transform methods and their applications in mapping mantle reflectivity structure. Surveys in Geophysics 30, 327-354.

Gudmundsson, Ó., Sambridge, M., 1998. A regionalized upper mantle (RUM) seismic model. Journal of Geophysical Research 103, 7121-7136.

Haupt, R.L., Haupt, S.E., 2004. Practical Genetic Algorithm, Second Edition. John Wiley \& Sons, New Jersey. 
Hayes, G.P., Wald, D.J., Johnson, R.L., 2012. Slab1.0: A three-dimensional model of global subduction zone geometries. Journal of Geophysical Research 117. doi:10.1029/2011JB008524.

Heit, B., Yuan, X., Bianchi, M., Kind, R., Gossler, J., 2010. Study of the lithospheric and upper-mantle discontinuities beneath eastern Asia by SS precursors. Geophysical Journal International 183, 252-266.

Honda, S., Morishige, M., Orihashi, Y., 2007. Sinking hot anomaly trapped at the $410 \mathrm{~km}$ discontinuity near the Honshu subduction zone, Japan. Earth and Planetary Science Letters 261, 565-577.

Houser, C., Williams, Q., 2010. Reconciling Pacific 410 and 660km discontinuity topography, transition zone shear velocity patterns, and mantle phase transitions. Earth and Planetary Science Letters 296, 255-266.

Huang, J., Zhao, D., 2006. High-resolution mantle tomography of China and surrounding regions. Journal of Geophysical Research 111:B09305. doi:10.1029/2005JB004066.

Ichiki, M., Baba, K., Obayashi, M., Utada, H., 2006. Water content and geotherm in the upper mantle above the stagnant slab: Interpretation of electrical conductivity and seismic P-wave velocity models. Physics of the Earth and Planetary Interiors 155, 1-15.

Ito, E., Takahashi, E., 1989. Postspinel transformations in the system $\mathrm{Mg}_{2} \mathrm{SiO}_{4}$ $\mathrm{Fe}_{2} \mathrm{SiO}_{4}$ and some geophysical implications. Journal of Geophysical Research 94, 10637-10646.

Jackson, M.G., Dasgupta, R., 2008. Compositions of HIMU, EM1, and EM2 from global trends between radiogenic isotopes and major elements in ocean island basalts. Earth and Planetary Science Letters 276, 175-186.

Jordan, T., 1977. Lithospheric slab penetration into the lower mantle beneath the Sea of Okhotsk. Journal of Geophysics 43, 473-496. 
852 Katsura, T., Ito, E., 1989. The system $\mathrm{Mg}_{2} \mathrm{SiO}_{4}-\mathrm{Fe}_{2} \mathrm{SiO}_{4}$ at high pressures and temperatures: Precise determination of stabilities of olivine, modified spinel, and spinel. Journal of Geophysical Research 94, 15663-15670.

Kuritani, T., Kimura, J.I., Miyamoto, T., Wei, H., Shimano, T., Maeno, F., Jin, upwelling asthenospheric mantle: Implications from the Changbaishan shield basalts, northeast China. Lithos 112, 247-258.

Larson, R., 1991. Latest pulse of Earth: Evidence for a mid-Cretaceous superplume. Geology 19, 547-550.

Laske, G., Masters, G., Ma, Z., Pasyanos, M., 2013. Update on CRUST1.0 - A 1degree global model of Earth's crust 15, Abstract EGU2013-2658.

Lawrence, J.F., Shearer, P.M., 2006a. Constraining seismic velocity and density for the mantle transition zone with reflected and transmitted waveforms. Geochemistry, Geophysics, Geosystems 7. doi:10.1029/2006GC001339.

Lawrence, J.F., Shearer, P.M., 2006b. A global study of transition zone thickness using receiver functions. Journal of Geophysical Research 111:B06307. doi:10.1029/2005JB003973.

Lebedev, S., van der Hilst, R.D., 2008. Global upper-mantle tomography with the automated multimode inversion of surface and S-wave forms. Geophysical Journal International 173, 505-518.

Lebedev, S., Nolet, G., 2003. Upper mantle beneath Southeast Asia from S velocity tomography. Journal of Geophysical Research: Solid Earth 108.

Lei, J., Zhao, D., 2005. P-wave tomography and origin of the Changbai intraplate volcano in Northeast Asia. Tectonophysics 397, 281-295.

Lessing, S., Thomas, C., Rost, S., Cobden, L., Dobson, D.P., 2014. Mantle transition zone structure beneath India and Western China from migration of PP and SS precursors. Geophysical Journal International 197, 396-413. 
879 Lessing, S., Thomas, C., Saki, M., Schmerr, N., Vanacore, E., 2015. On the 880 difficulties of detecting PP precursors. Geophysical Journal International 201, $881 \quad 1666-1681$.

Li, C., van der Hilst, R.D., 2010. Structure of the upper mantle and transition zone beneath Southeast Asia from traveltime tomography. Journal of Geophysical Research 115. doi:10.1029/2009JB006882.

Li, C., van der Hilst, R.D., Engdahl, E.R., Burdick, S., 2008. A new global model for P wave speed variations in Earth's mantle. Geochemistry, Geophysics, Geosystems 9. doi:10.1029/2007GC001806.

Li, X., Kind, R., Yuan, X., 2003. Seismic study of upper mantle and transition zone beneath hotspots. Physics of the Earth and Planetary Interiors 136, 7992.

Li, X., Sobolev, S., Kind, R., Yuan, X., Estabrook, C., 2000. A detailed receiver function image of the upper mantle discontinuities in the Japan subduction zone. Earth and Planetary Science Letters 183, 527-541.

Li, X., Yuan, X., 2003. Receiver functions in northeast China-implications for slab penetration into the lower mantle in northwest Pacific subduction zone. Earth and Planetary Science Letters 216, 679-691.

Liu, Q., Gu, Y., 2012. Seismic imaging: From classical to adjoint tomography. Tectonophysics 566, 31-66.

Liu, Z., Niu, F., Chen, Y.J., Grand, S., Kawakatsu, H., Ning, J., Tanaka, S., Obayashi, M., Ni, J., 2015. Receiver function images of the mantle transition zone beneath ne china: New constraints on intraplate volcanism, deep subduction and their potential link. Earth and Planetary Science Letters 412, 101-111.

Marone, F., Gung, Y., Romanowicz, B., 2007. Three-dimensional radial anisotropic structure of the North American upper mantle from inversion of surface waveform data. Geophysical Journal International 171, 206-222. 
Mégnin, C., Romanowicz, B., 2000. The three-dimensional shear velocity structure of the mantle from the inversion of body, surface and higher-mode waveforms. Geophysical Journal International 143, 709-728.

Navrotsky, A., 1980. Lower mantle phase transitions may generally have negative pressure-temperature slopes. Geophysical Research Letters 7, 709-711.

Niu, Y., 2005. Generation and evolution of basaltic magmas: some basic concepts and a new view on the origin of Mesozoic-Cenozoic basaltic volcanism in eastern China. Geological Journal of Chine Universities 11, 9-46.

Obayashi, M., Sugioka, H., Yoshimitsu, J., Fukao, Y., 2006. High temperature anomalies oceanward of subducting slabs at the $410-\mathrm{km}$ discontinuity. Earth and Planetary Science Letters 243, 149-158.

Ohtani, E., Litasov, K., Hosoya, T., Kubo, T., Kondo, T., 2004. Water transport into the deep mantle and formation of a hydrous transition zone. Physics of the Earth and Planetary Interiors 143, 255-269.

Priestley, K., Debayle, E., McKenzie, D., Pilidou, S., 2006. Upper mantle structure of eastern Asia from multimode surface waveform tomography. Journal of Geophysical Research: Solid Earth 111 (B10).

Revenaugh, J., Jordan, T.H., 1991. Mantle layering from ScS reverberations: 2. The transition zone. Journal of Geophysical Research 96, 19763-19780.

Ritsema, J., Deuss, A., Van Heijst, H., Woodhouse, J., 2011. S40RTS: a degree40 shear-velocity model for the mantle from new Rayleigh wave dispersion, teleseismic traveltime and normal-mode splitting function measurements. Geophysical Journal International 184, 1223-1236.

Rost, S., Thomas, C., 2009. Improving seismic resolution through array processing techniques. Surveys in Geophysics 30, 271-299.

Sacchi, M.D., 1998. A bootstrap procedure for high-resolution velocity analysis. Geophysics 63, 1716-1725. 
934 Schmandt, B., Humphreys, E., 2010. Complex subduction and small-scale convection revealed by body-wave tomography of the western United States upper mantle. Earth and Planetary Science Letters 297, 435-445.

Schmerr, N., Garnero, E., 2006. Investigation of upper mantle discontinuity structure beneath the central Pacific using SS precursors. Journal of Geophysical Research 111:B08305. doi:10.1029/2005JB004197.

Schmerr, N., Garnero, E.J., 2007. Upper mantle discontinuity topography from thermal and chemical heterogeneity. Science 318, 623-626.

Schmerr, N., Thomas, C., 2011. Subducted lithosphere beneath the Kuriles from migration of PP precursors. Earth and Planetary Science Letters 311, 101-111.

Shearer, P.M., 1991. Constraints on upper mantle discontinuities from observations of long-period reflected and converted phases. Journal of Geophysical Research 96, 18147-18182.

Shearer, P.M., 1993. Global mapping of upper mantle reflectors from long-period SS precursors. Geophysical Journal International 115, 878-904.

Shearer, P.M., Flanagan, M.P., Hedlin, M.A., 1999. Experiments in migration processing of SS precursor data to image upper mantle discontinuity structure. Journal of Geophysical Research: Solid Earth 104, 7229-7242.

Simmons, N.A., Forte, A.M., Boschi, L., Grand, S.P., 2010. GyPSuM: A joint tomographic model of mantle density and seismic wave speeds. Journal of Geophysical Research: Solid Earth 115.

Stoffa, P.L., Sen, M.K., 1991. Nonlinear multiparameter optimization using genetic algorithms: Inversion of plane-wave seismograms. Geophysics 56, 1794-1810.

Tang, Y., Obayashi, M., Niu, F., Grand, S.P., Chen, Y.J., Kawakatsu, H., Tanaka, S., Ning, J., Ni, J.F., 2014. Changbaishan volcanism in northeast China linked to subduction-induced mantle upwelling. Nature Geoscience 7, 470-475. 
961 Tatsumi, Y., Maruyama, S., Nohda, S., 1990. Mechanism of backarc opening in the Japan Sea: role of asthenospheric injection. Tectonophysics 181, 299-306. Tatsumi, Y., Shinjoe, H., Ishizuka, H., Sager, W.W., Klaus, A., 1998.

Geochemical evidence for a mid-Cretaceous superplume. Geology 26, 151154.

Tauzin, B., Debayle, E., Wittlinger, G., 2008. The mantle transition zone as seen by global Pds phases: no clear evidence for a thin transition zone beneath hotspots. Journal of Geophysical Research: Solid Earth 113:B08309.

van der Hilst, R., Engdahl, E., Spakman, W., 1993. Tomographic inversion of P and $\mathrm{pP}$ data for aspherical mantle structure below the northwest Pacific region. Geophysical Journal International 115, 264-302.

van der Hilst, R., Engdahl, E., Spakman, W., Nolet, G., 1991. Tomographic imaging of subducted lithosphere below northwest Pacific island arcs. Nature $353,37-42$.

Weidner, D.J., Wang, Y., 1998. Chemical-and Clapeyron-induced buoyancy at the $660 \mathrm{~km}$ discontinuity. Journal of geophysical research 103, 7431-7441.

Ye, L., Li, J., Tseng, T.L., Yao, Z., 2011. A stagnant slab in a waterbearing mantle transition zone beneath northeast China: implications from regional SH waveform modelling. Geophysical Journal International 186, 706-710.

Yuan, H., Romanowicz, B., 2010. Lithospheric layering in the North American craton. Nature 466, 1063-1068.

Zhao, D., 2007. Seismic images under 60 hotspots: Search for mantle plumes. Gondwana Research 12, 335-355.

Zhao, D., Lei, J., Tang, R., 2004. Origin of the Changbai intraplate volcanism in Northeast China: evidence from seismic tomography. Chinese Science Bulletin 49, 1401-1408.

Zhao, D., Liu, L., 2010. Deep structure and origin of active volcanoes in China. Geoscience Frontiers 1, 31-44. 
989 Zhao, D., Ohtani, E., 2009. Deep slab subduction and dehydration and their 990 geodynamic consequences: evidence from seismology and mineral physics. 991 Gondwana Research 16, 401-413.

992 Zhao, D., Yanada, T., Hasegawa, A., Umino, N., Wei, W., 2012. Imaging the 993 subducting slabs and mantle upwelling under the Japan Islands. Geophysical 994 Journal International 190, 816-828.

995 Zheng, Z., Romanowicz, B., 2012. Do double SS precursors mean double 996 discontinuities? Geophysical Journal International 191, 1361-1373.

997 


\section{List of Figures}

999

1000 Figure 1: (a) Global distribution of the earthquake (red stars) and station (blue triangles) locations

1001 used in this study. (b) Distribution of the $S S$ bouncepoints from the earthquake-station pairs. The

1002 convergent plate boundaries and slab contours are indicated by the red and blue lines,

1003 respectively. The contour lines are taken at a constant interval of $50 \mathrm{~km}$ starting at $100 \mathrm{~km}$ depth (Hayes et al., 2012). The SS precursor waveforms are stacked into 30 bins along three parallel

1005 profiles A, B and C. (c) Path coverage of the SS precursors that are used in the stacking and inversion procedures. (d) A graphical representation of theoretical ray paths of $S S$ and its precursors for a source-receiver distance of 130.7 degrees. The left panel shows an observed seismogram aligned on the maximum amplitude of the SS phase. S410S and S660 are marked based on the predicted arrival times of PREM (Dziewonski and Anderson, 1981). .7

Figure 2: (a) An input transverse component seismogram with $10 \%$ noise (gray) and the inverted trace after 15 generations (black). The precursors are magnified by a factor of 3 and the difference (data-synthetic) seismogram has been offset by -0.08 for clarity. (b) The input (gray) and the recovered (black) model velocities. The red lines indicate the lower and upper boundaries of the model space. (c) The best and mean values of the objective function in each generation. (d) Colorcoded velocity output from nonlinear inversion of the noise-added synthetic seismogram. Random input velocity structures are assumed at the first generation.

Figure 3: The interpolated reflectivity maps of $S 410 S$ (a) and $S 660 S$ (b) amplitude variations. (c) and (d) show the standard errors of the reflection amplitudes based on a bootstrapping analysis. The solid circles denote the centers of the averaging bins and the magenta lines show the slab contours (Hayes et al., 2012). The numbers at the center locations of the averaging bins in panel red lines represent the observed and synthetic stacks, respectively. The key features in the waveform data are highlighted using the dashed boxes. The symbols D and E represent areas with depressed and elevated discontinuity topography, respectively. The letters $\mathrm{H}$ and $\mathrm{L}$ indicate the 
1028

1029

1030

1031

1032

1033

1034

1035

1036

1037

1038

1039

1040

1041

1042

1043

1044

1045

1046

1047

1048

1049

1050

1051

1052

1053

1054

1055

1056

1057

1981) at $300 \mathrm{~km} \mathrm{(a),} 400 \mathrm{~km}$ (b), $500 \mathrm{~km}$ (c), and $600 \mathrm{~km}$ (d) depths beneath NE China and the northwestern Pacific region. The blue and red colors represent fast and slow anomalies, respectively. The solid circles mark the centers of the averaging caps and the white triangles denote the locations of major intraplate volcanoes.

Figure 6: (a) MTZ thickness variations inferred from the inverted depths of the 410 and 660 by this study. (b) MTZ thickness variations reported by Gu and Dziewonski (2002). The red and blue colors represent regions of narrow and thick MTZ, respectively. Z1 and Z2 indicate anomalously thick MTZ beneath NE China and the Ryukyu trench, respectively. The dashed-line box in panel (a) outlines a relatively thick MTZ (>270 km) in Liu et al. (2015), obtained from depth migrated P-to-S converted waves.

Figure 7: (a)-(c) West-east vertical cross-sections of the inverted shear wave velocity anomalies along profiles A to $\mathrm{C}$ from 200 to $720 \mathrm{~km}$ depth beneath the northwestern Pacific subduction zones and NE China. The magenta circles indicate the locations of deep earthquakes within a $2^{\circ}$ distance from each profile. The depths of the 410 and $660 \mathrm{~km}$ discontinuities are indicated by the dashed lines. A1 and A2 mark the downgoing and stagnant parts of the Pacific slab, respectively, while A3 and A4 denote low shear velocity anomalies beneath the Changbai-Wudalianchi hotspot. A5 indicates low seismic velocities on the ocean side of the Wadati-Benioff zone. (d)-(f) Similar to (a)-(c), but for an earlier model of $P$-wave velocities (Obayashi et al., 2006).

Figure 8: (a) A cross-section of our shear velocity model along profile C. The dashed and solid straight lines indicate how the MTZ thickness and average MTZ velocity are measured along slab dips of 30 and 45 degrees, respectively. (b) The correlation between MTZ thickness and the average MTZ shear velocity perturbations at a range of dip angles. (c) Observations of apparent MTZ thickness versus the apparent average MTZ shear velocity along slab dip of 30 degrees. The least-square fit and correlation coefficient are also shown. (d) Similar to (c) but for slab dip of 90 degrees.

Figure 9: Input models (panels a and b) and the inverted results (panels $\mathrm{c}$ and d) of the synthetic tests along profiles B and C. The depths of the 410 and 660 are indicated by the thick dashed lines. .26

Figure 10: 2D waveform tomography results using the stacked waveforms observed along profile B (see Figure 4). The results clearly show a high velocity structure, associated with the subducting 
1059 paths used in waveform tomography. The sources and receivers are indicated by stars and 1060 triangles, respectively. The thick black lines outline the area covered by the waveform 1061 tomography approach. The outlined area is zoomed on from $200 \mathrm{~km}$ to $750 \mathrm{~km}$ depth. (b) The 1062 visual comparison between the observed (black) and synthetic (red) waveforms shows a good 1063 agreement between the input and modeled waveforms. (c). The normalized fitness values of the 1064 objective function in each generation. (d) Color-coded cross-section (outlined area in panel a) of 1065 the shear wave velocity perturbations. The blue and red colors represent the fast and slow 1066 velocities, respectively. The key features of the recovered image are also labeled (LVZ stands for 1067 low velocity zone).

1068 Figure 11: (a) Isosurfaces showing the major seismic anomalies beneath Northeast China and the 1069 northwestern Pacific subduction zones in the depth range of 200 to $700 \mathrm{~km}$. The blue and red 1070 colors represent fast and slow shear velocities, respectively, at the respective isosurfaces of $1071+0.65 \%$ and $-1.0 \%$. The definitions of major seismic anomalies (A1-A5) are the same as in Figure

1072 7. The black lines show the slab contours, which are taken at $50 \mathrm{~km}$ depth intervals starting at 100 $1073 \mathrm{~km}$ (Hayes et al., 2012), and the magenta circles indicate the locations of deep-focus earthquakes. 1074 (b) A schematic illustration of a NW-SE vertical section (see Figure 11a) showing the possible 1075 origins of the intraplate volcanism in Northeast China. Three possible mechanisms for the 1076 intraplate volcanism (discussed in Section 4.3) are also illustrated: (1) deep dehydration process of 1077 the slab, (2) passive upwelling in response to the subduction of the Pacific plate, and (3) escaping 1078 of the hot sub-slab material through a slab window 
(a)

(b)
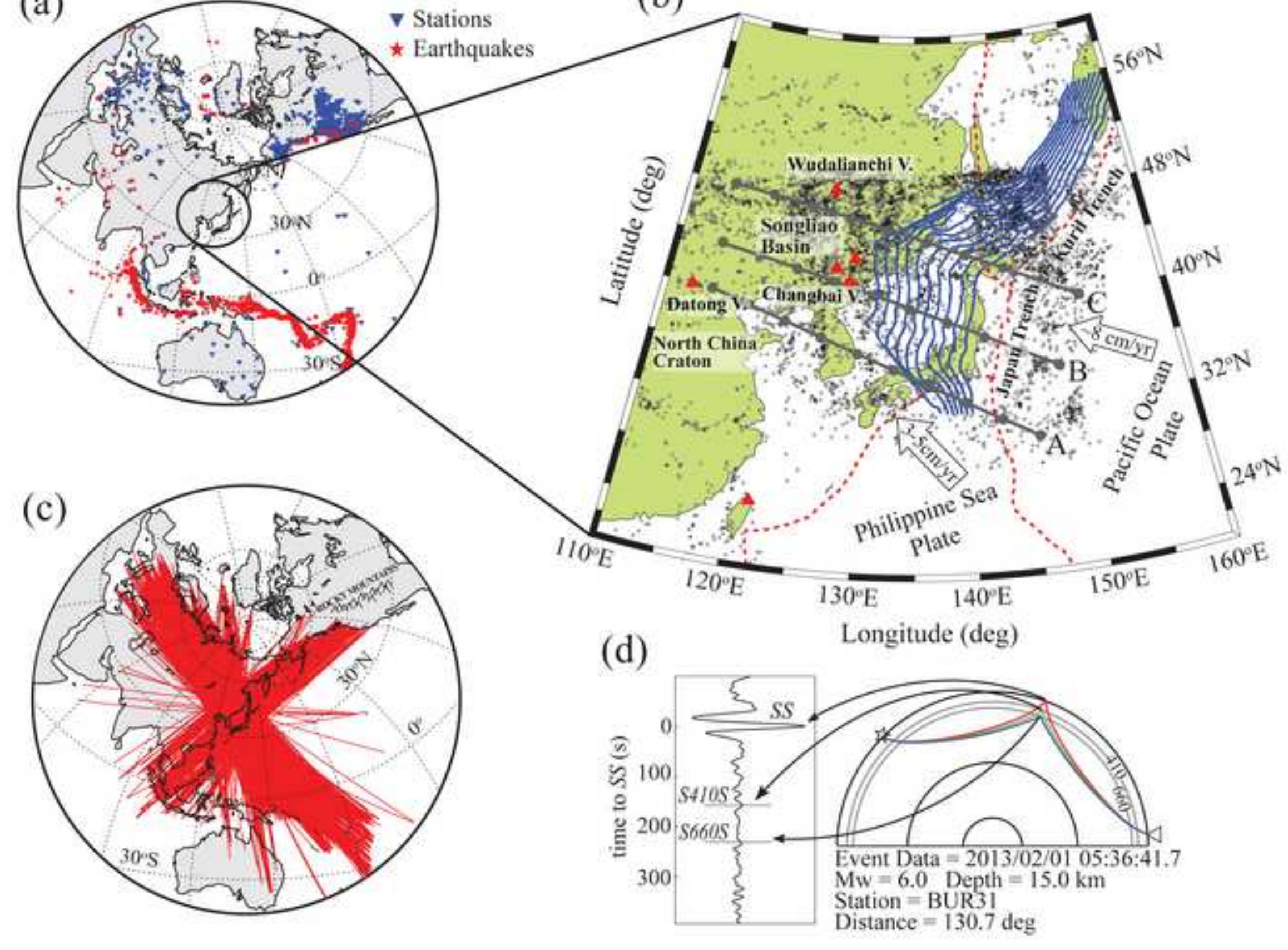
(a)
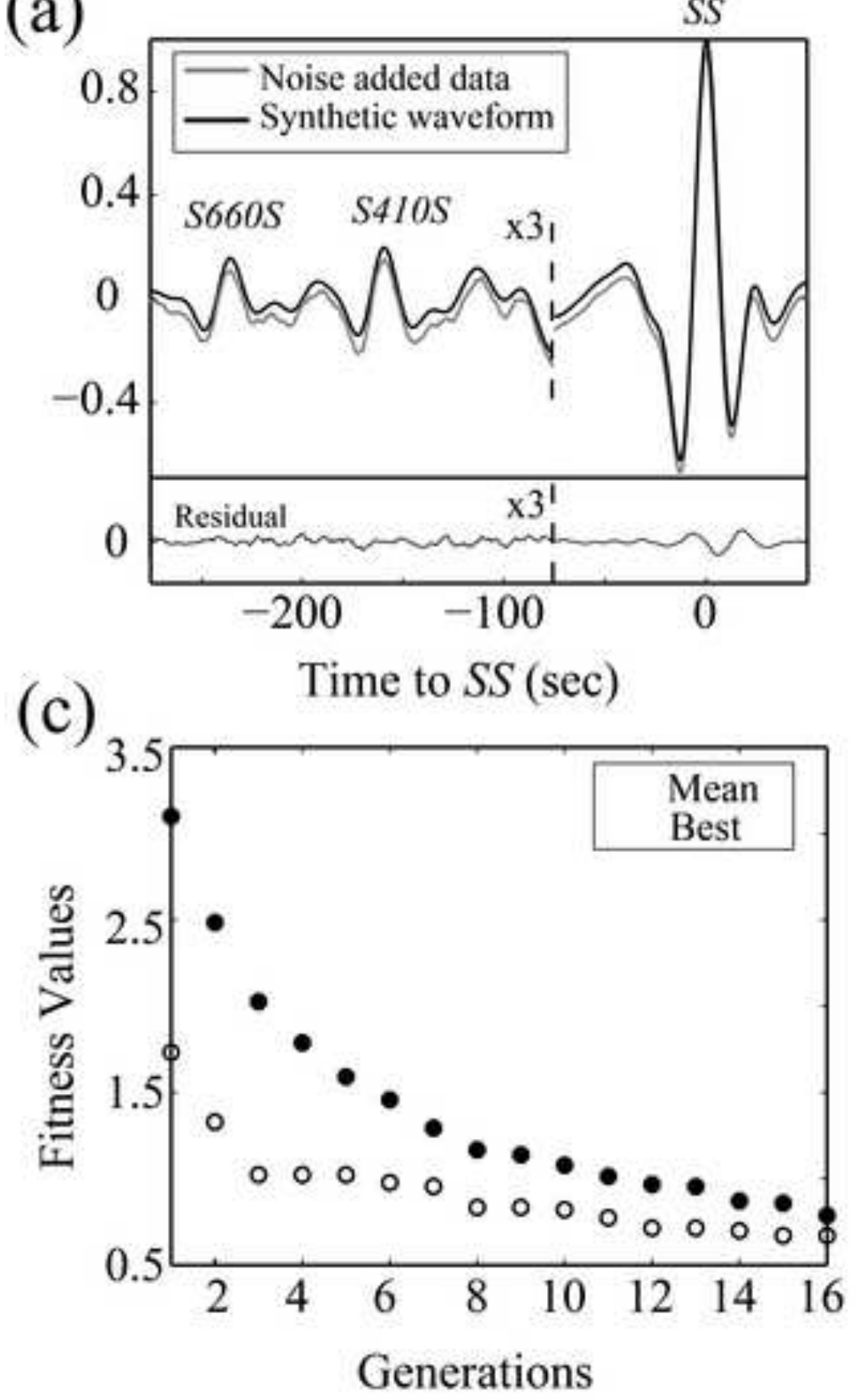

(b)

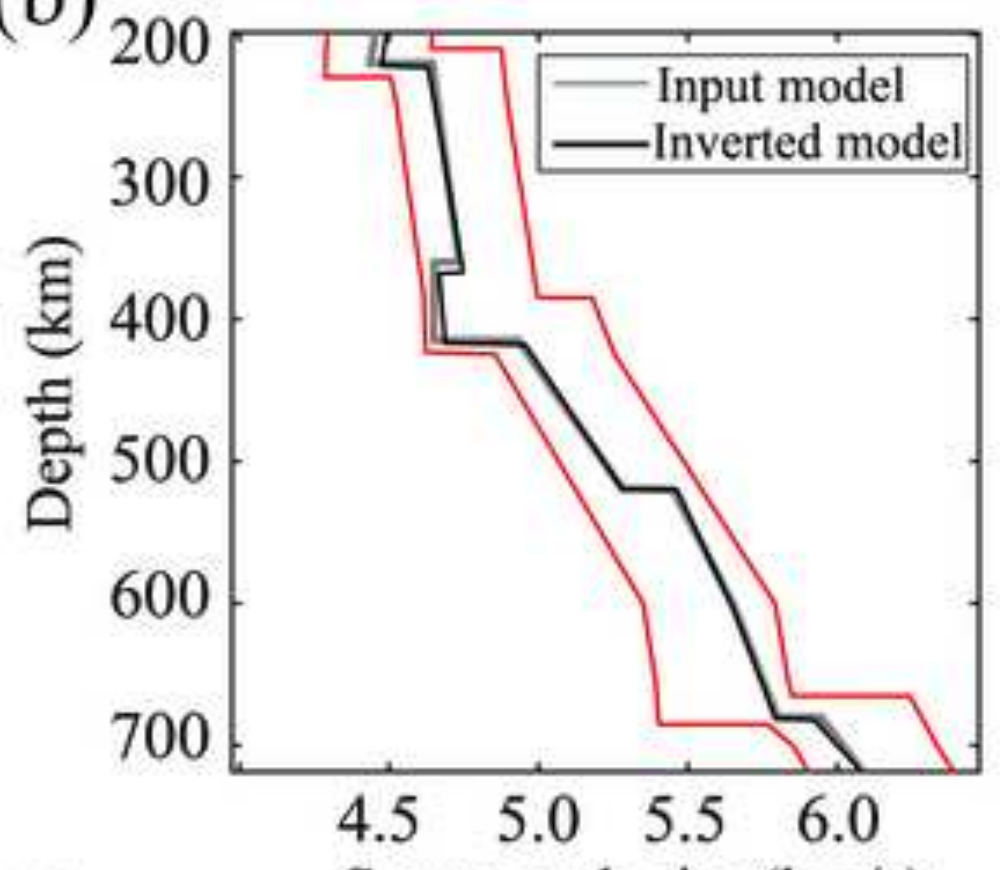

(d)

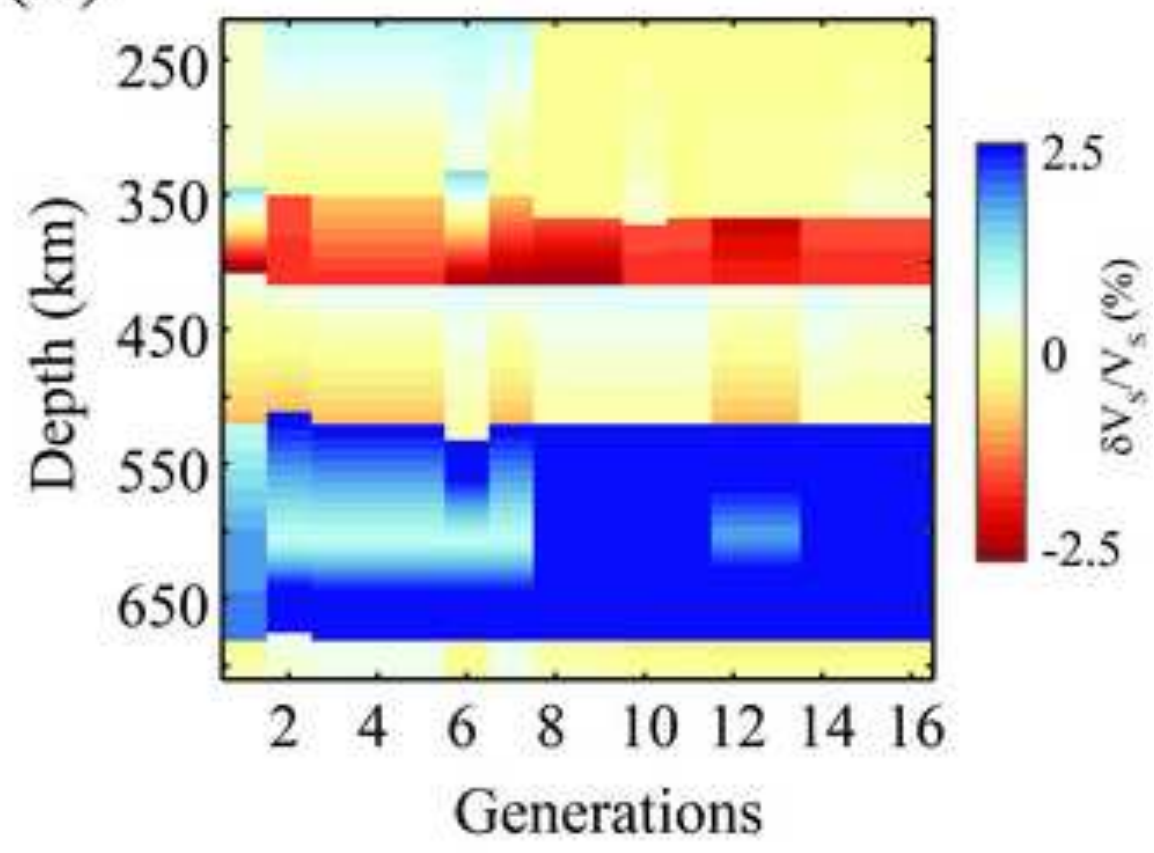




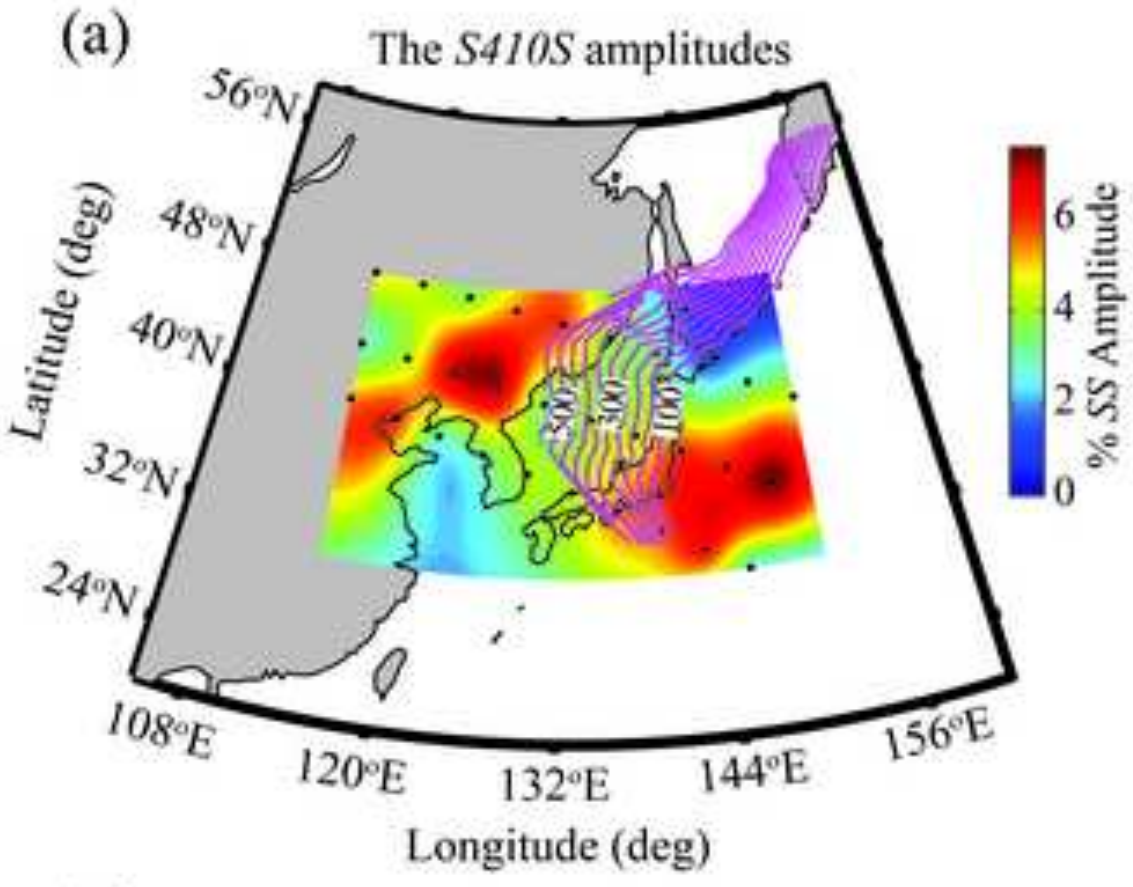

(c) The standard errors of the $\$ 410 S$ amplitudes

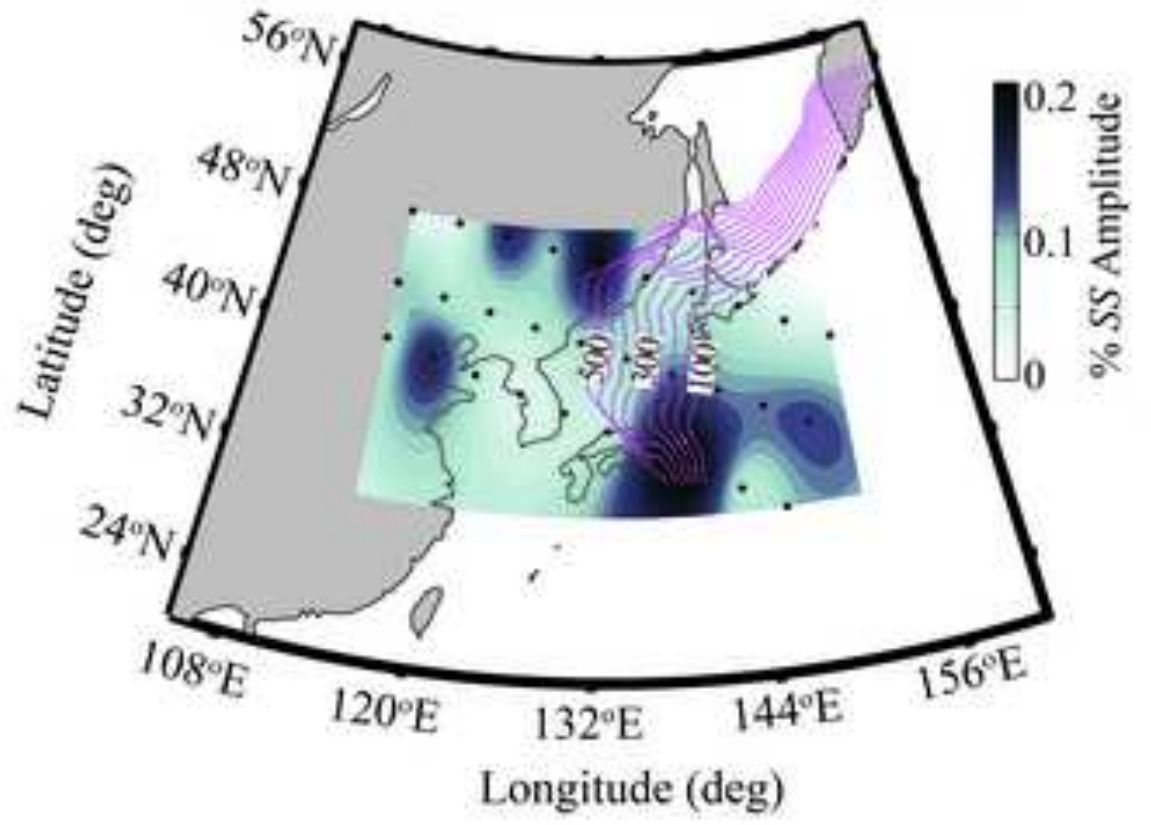

(b)

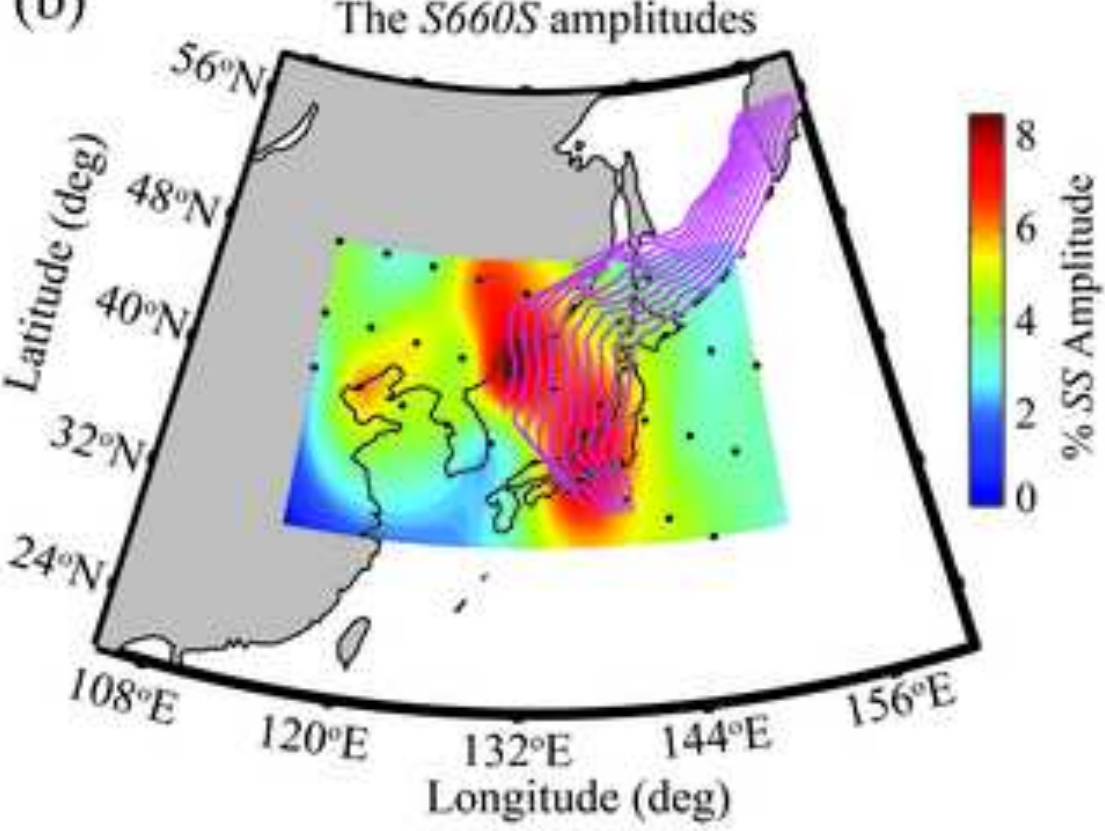

(d) The standard errors of the 56605 amplitudes

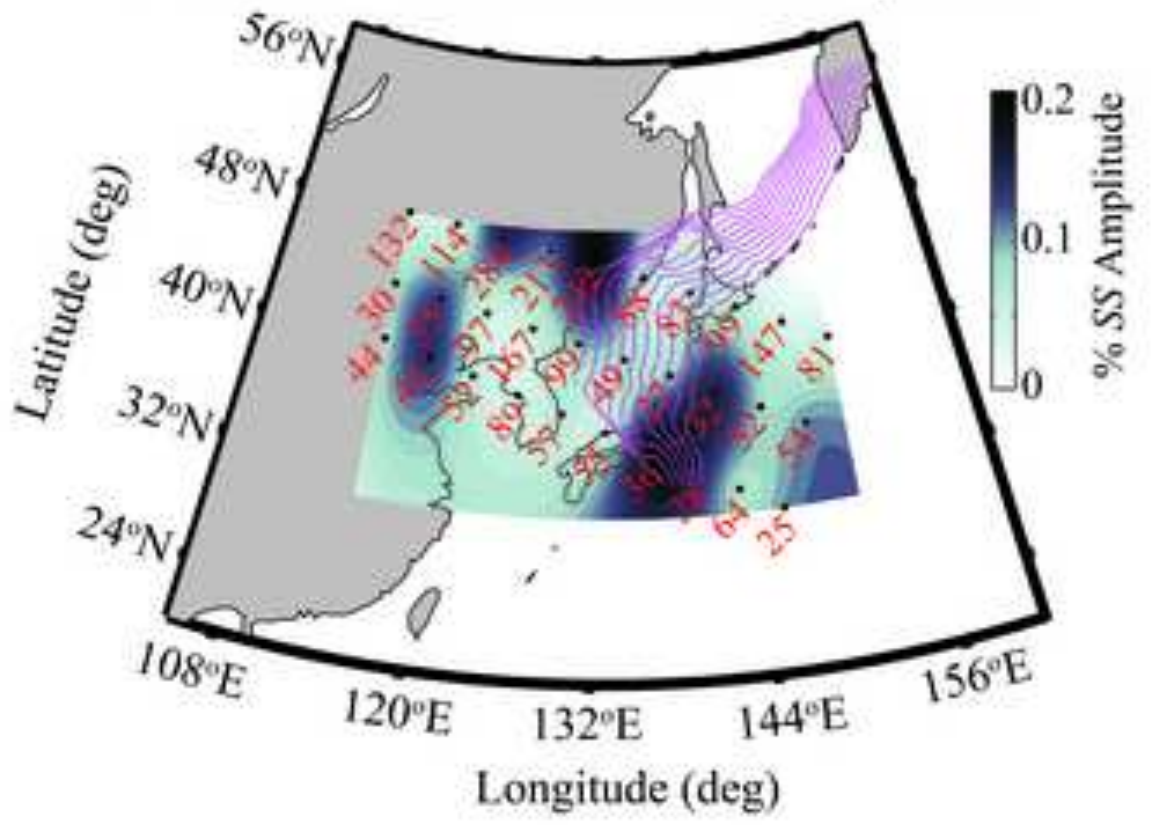


(a) Profile $\mathrm{A}$

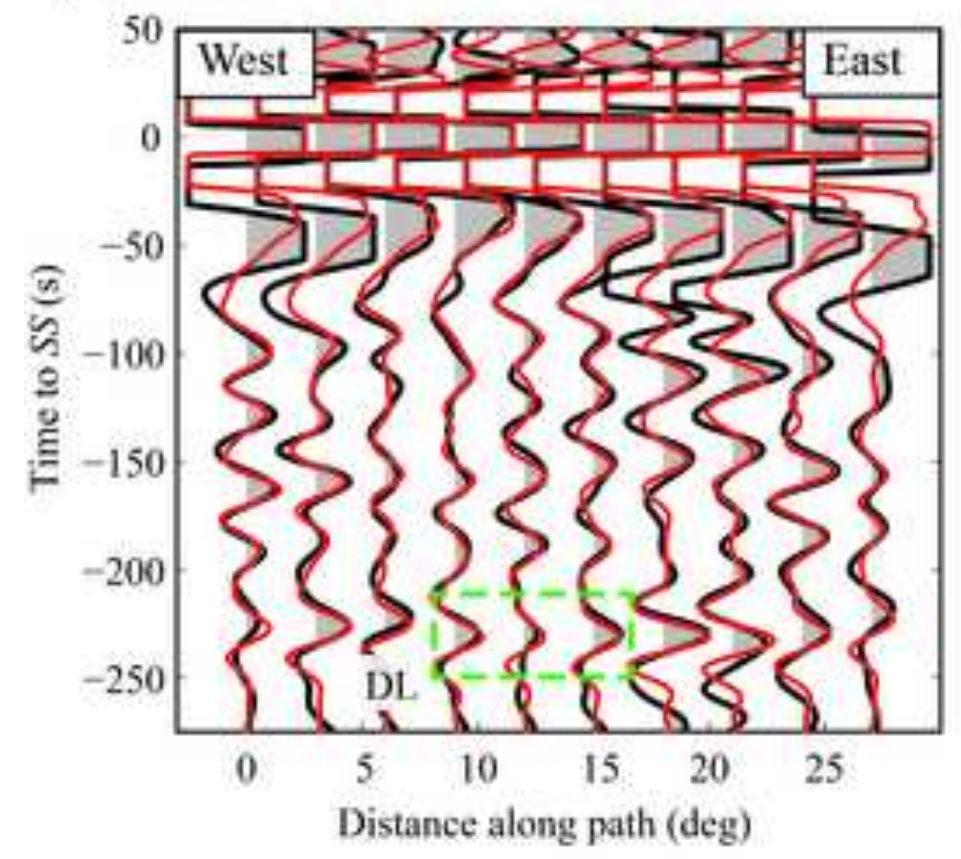

(b) Profile B

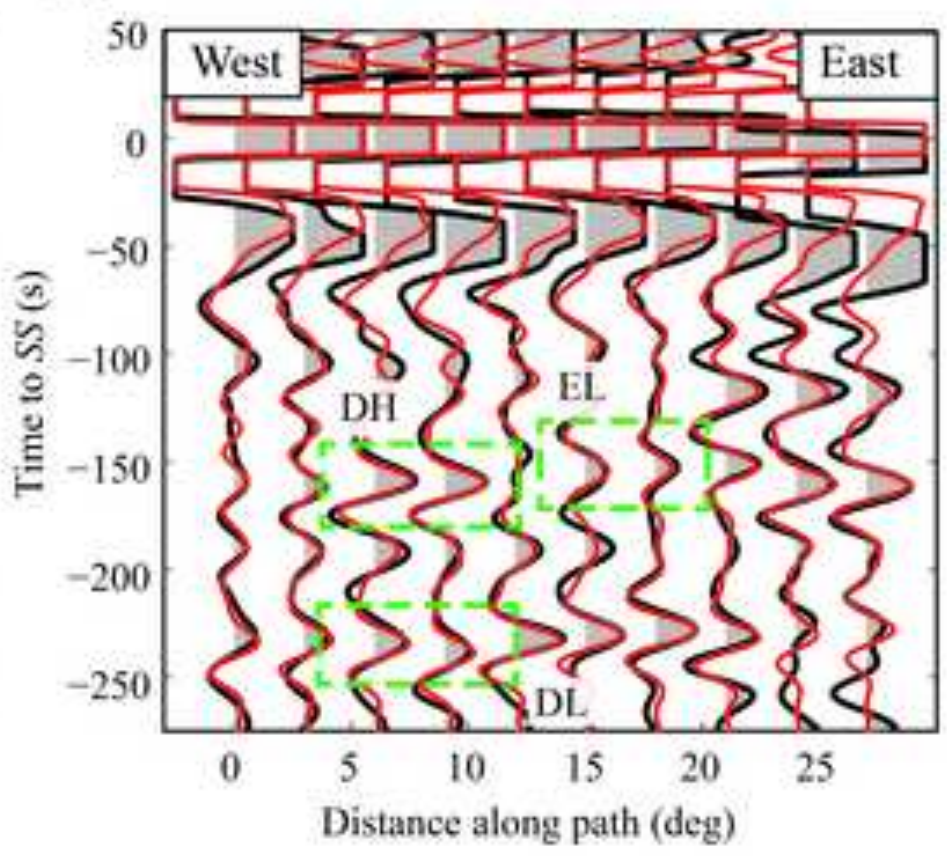

(c) Profile $\mathrm{C}$

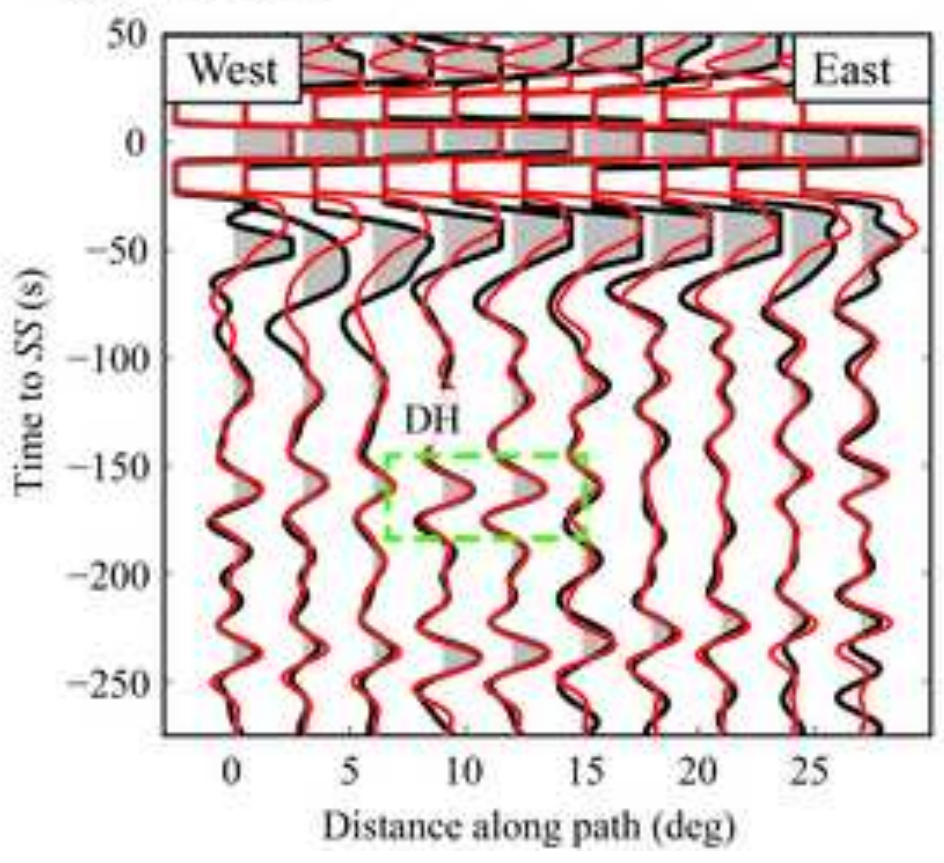


(a)

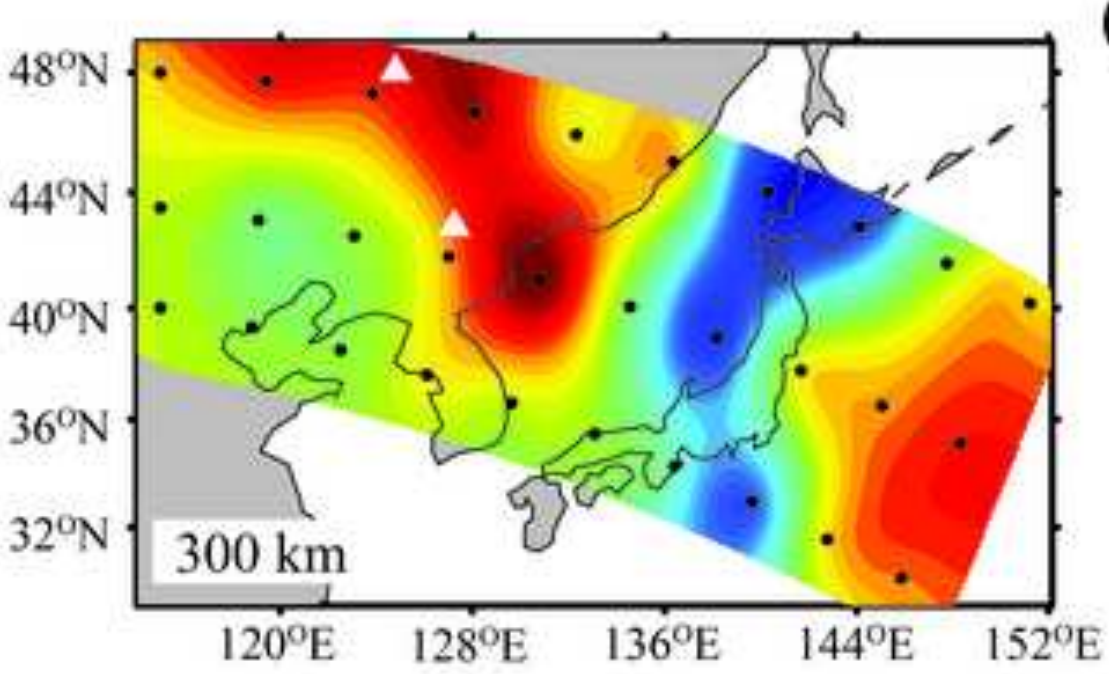

(c)

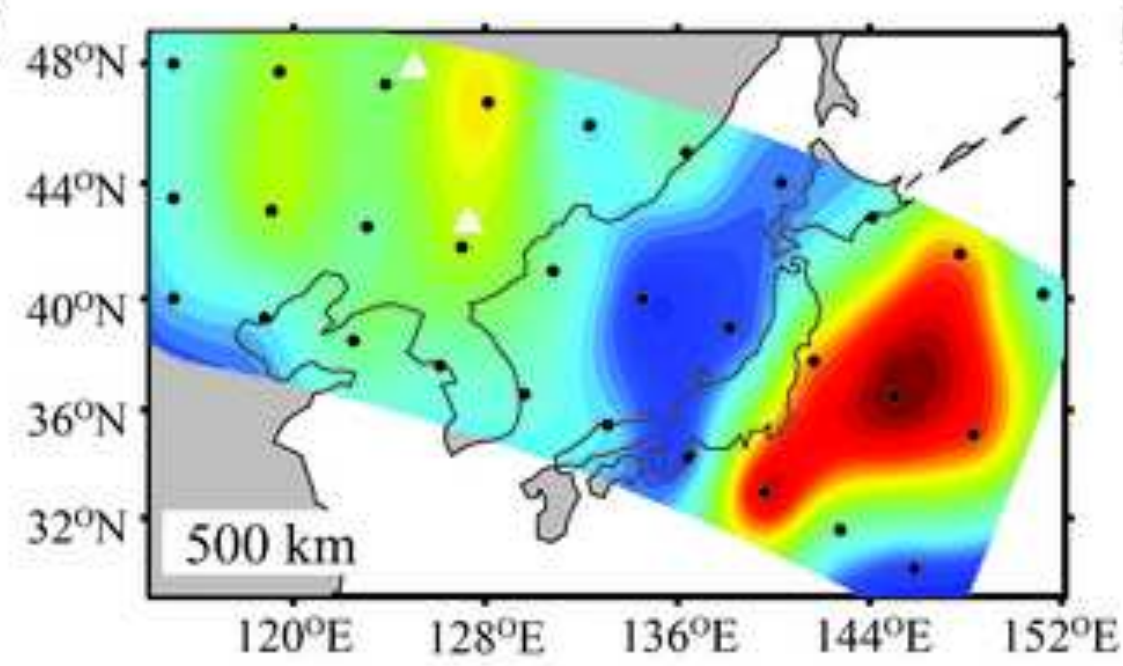

(b)

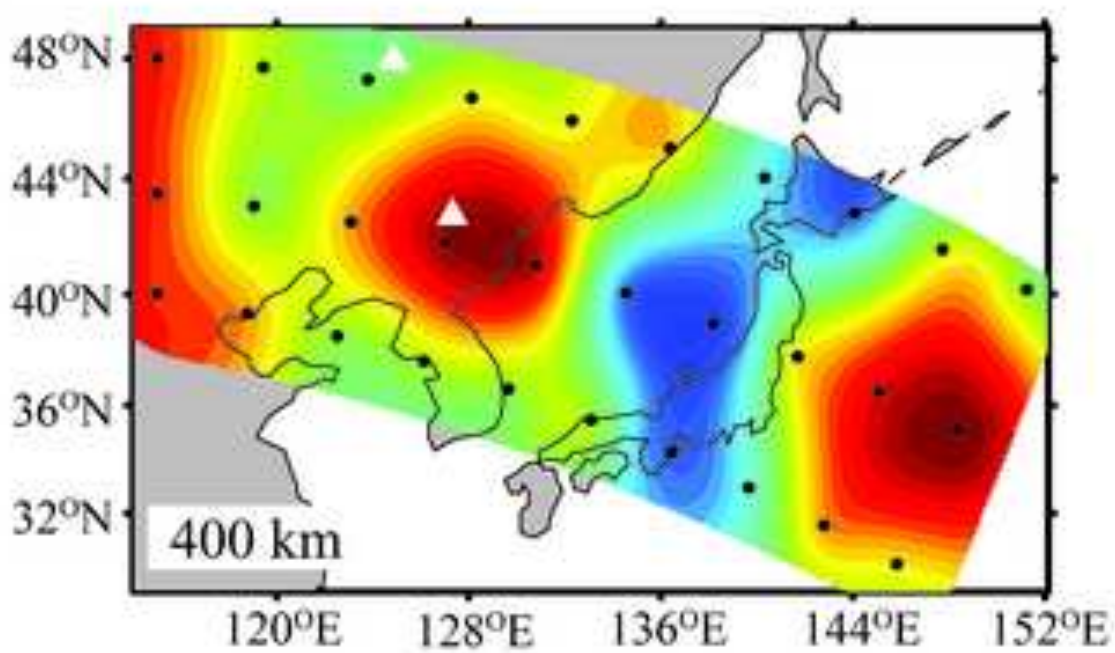

(d)

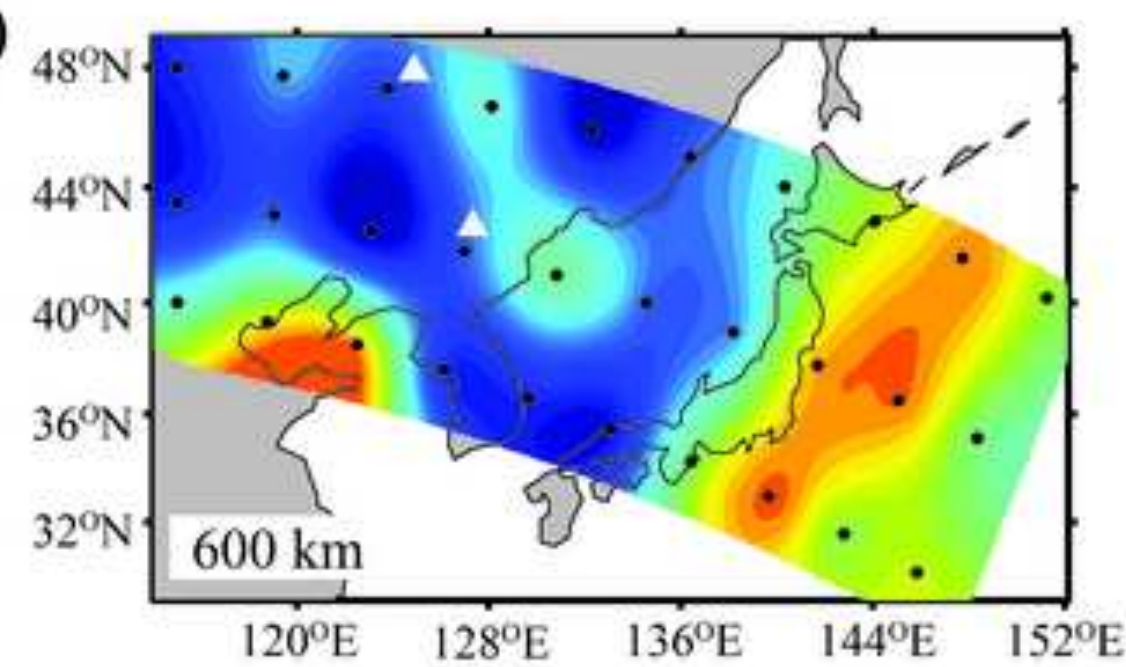

$$
-1 \underset{\delta \mathrm{V} / \mathrm{V}(\%)}{0} 1
$$


(a)

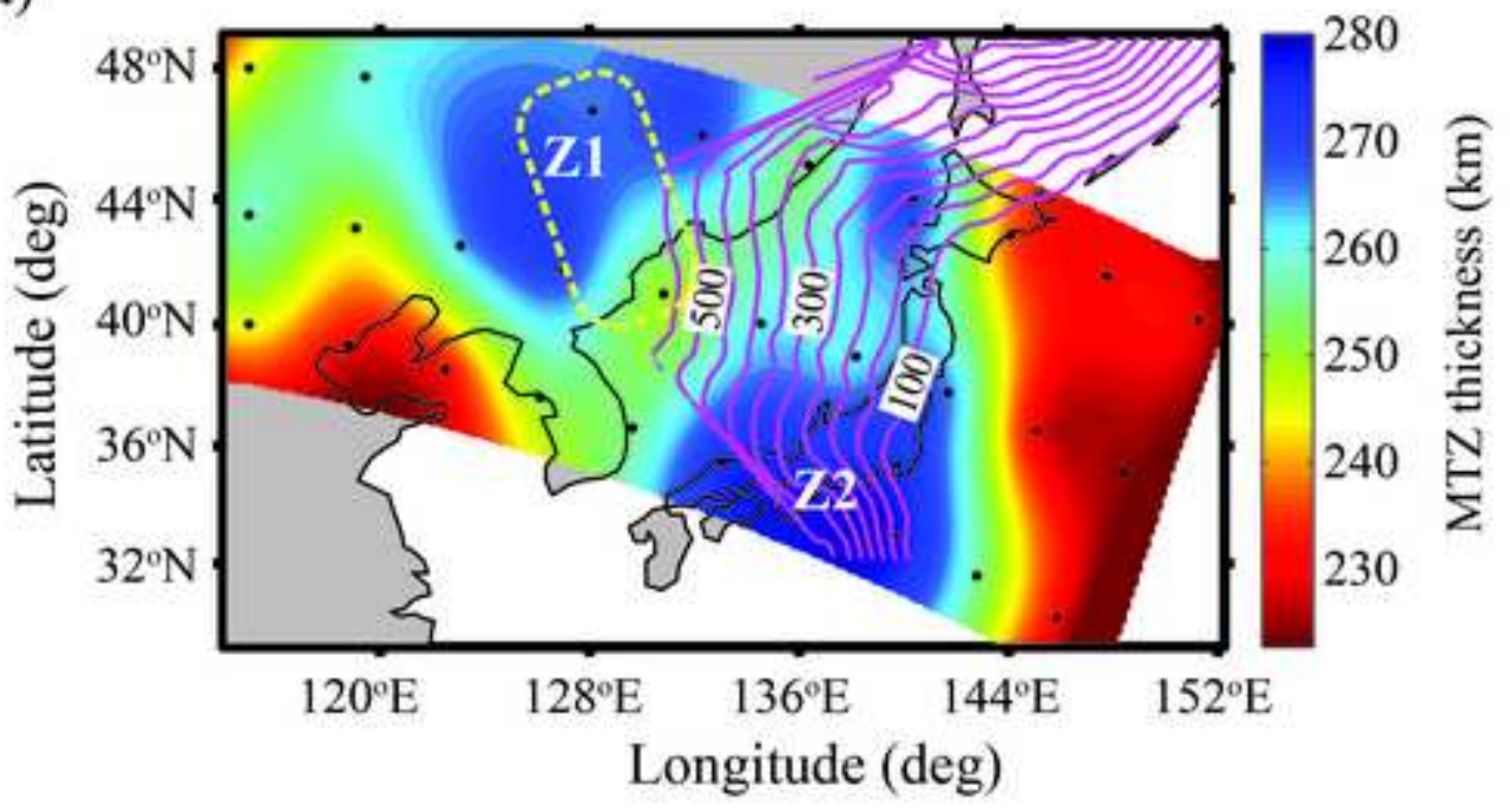

(b)

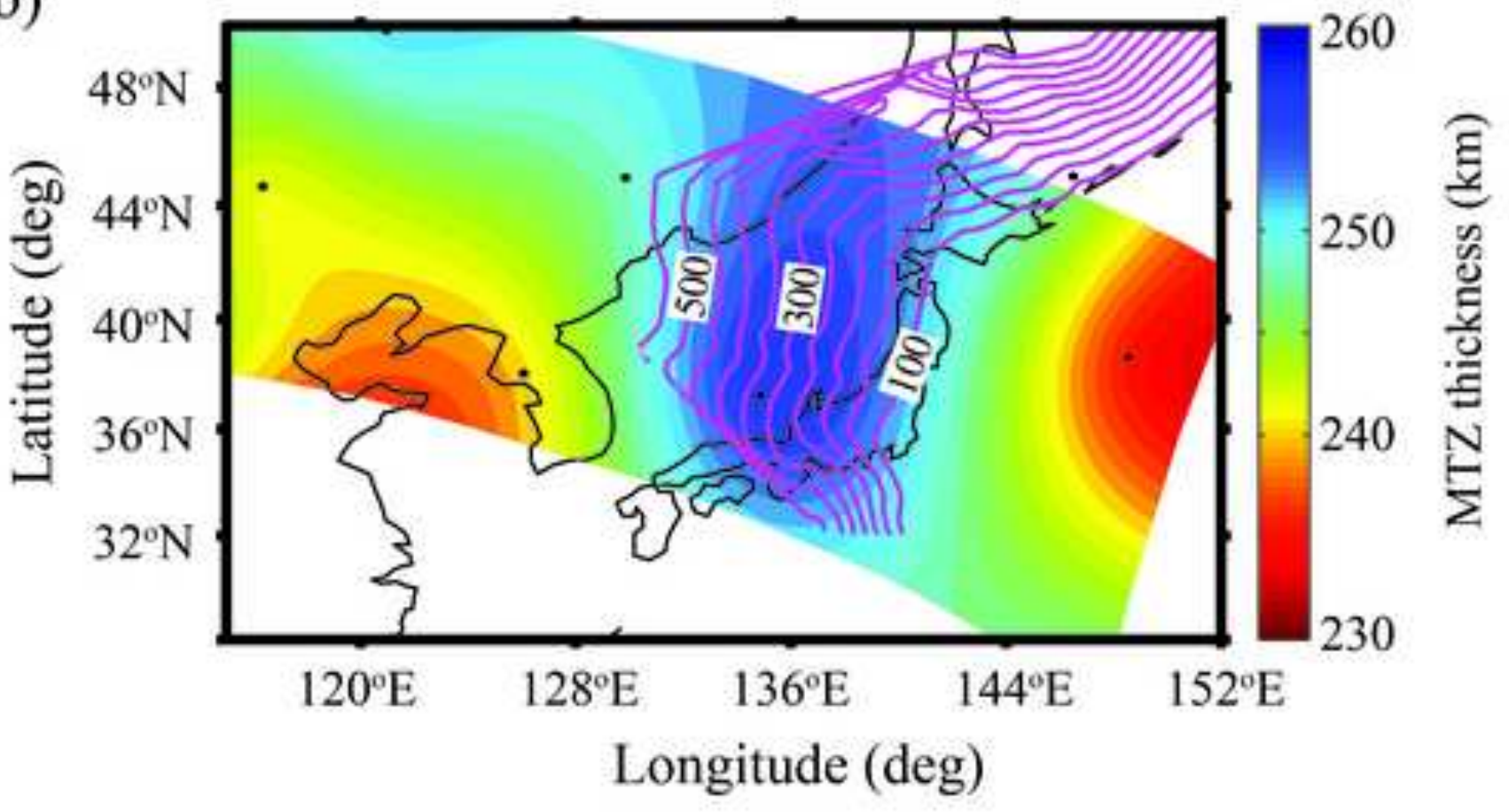


(a)

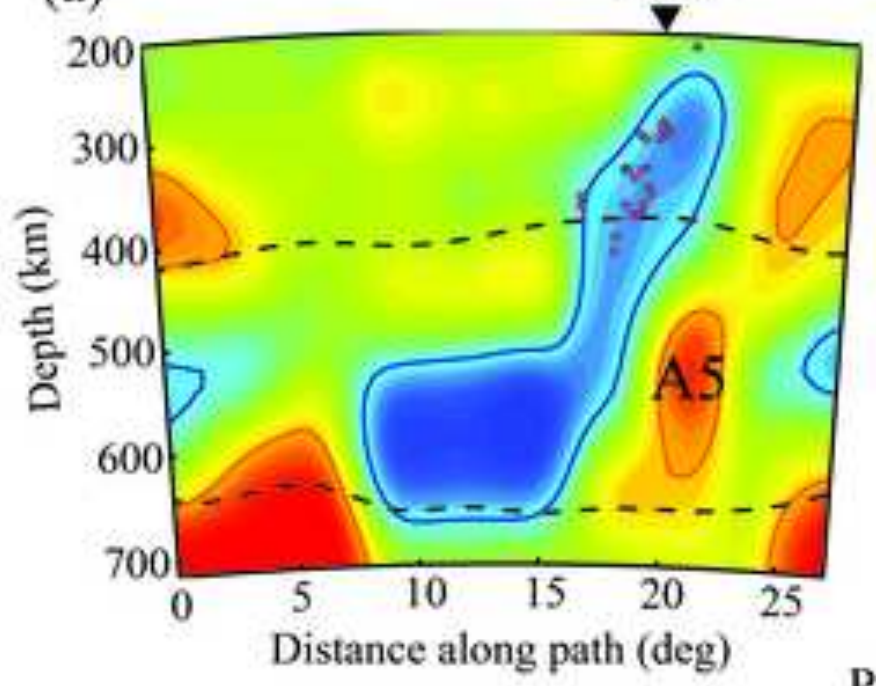

(b)

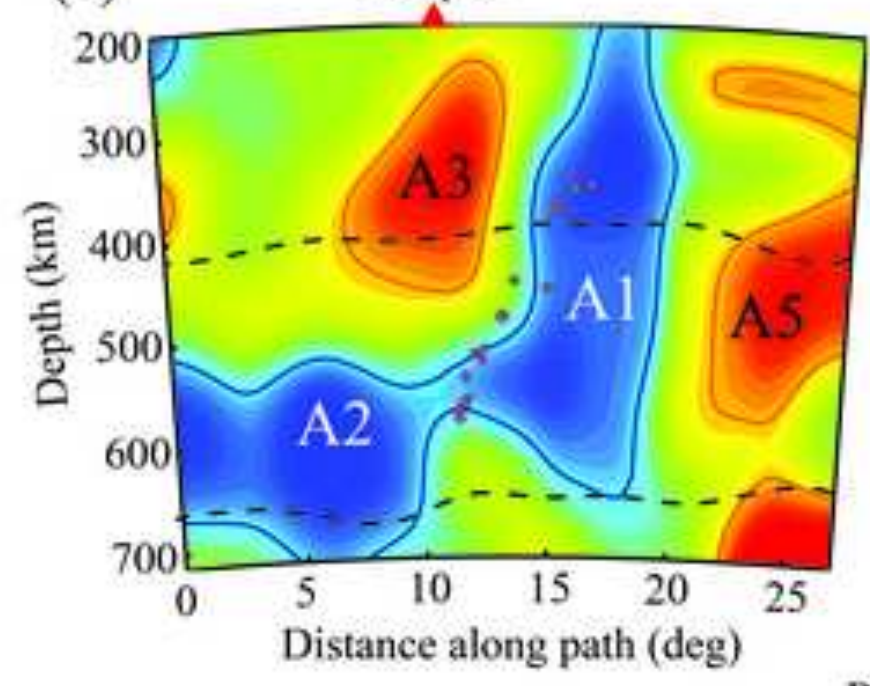

(c) Wudalianchi

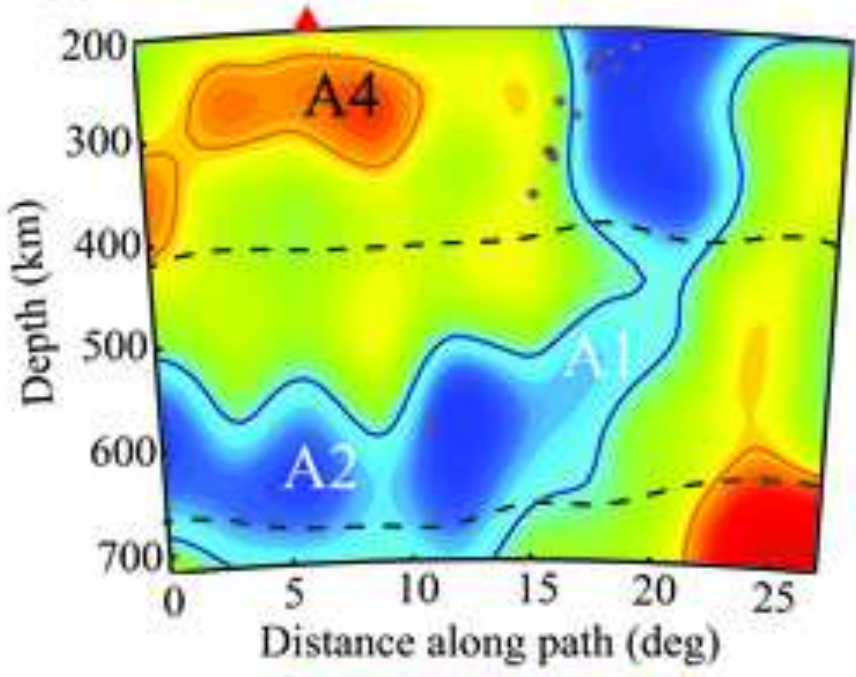

Profile B

(d)

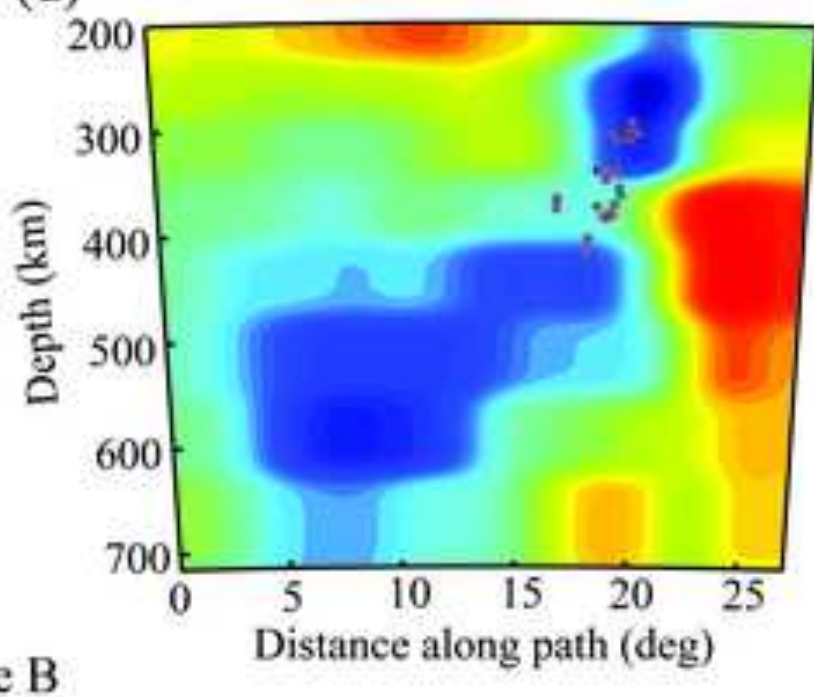

(e)

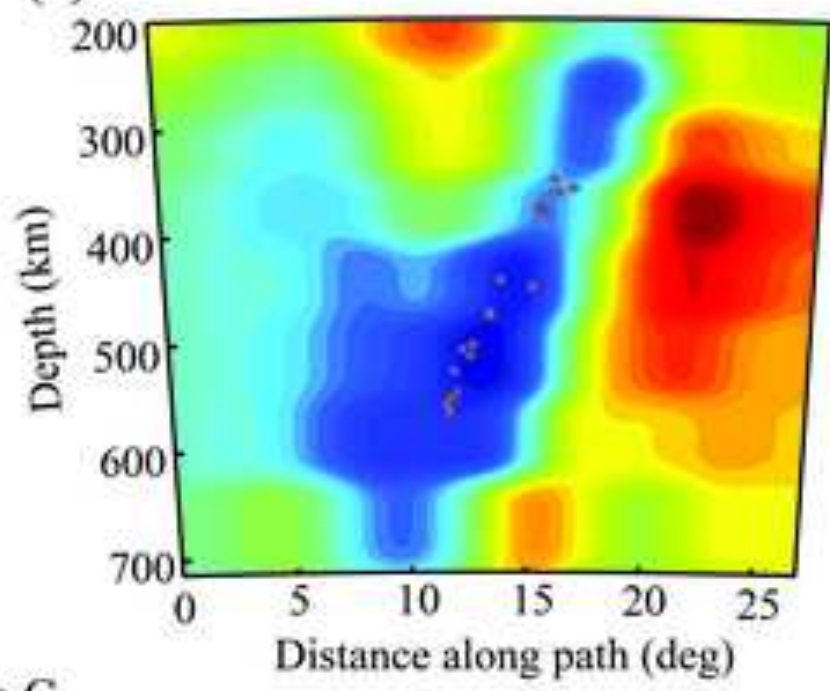

Profile C

(f)

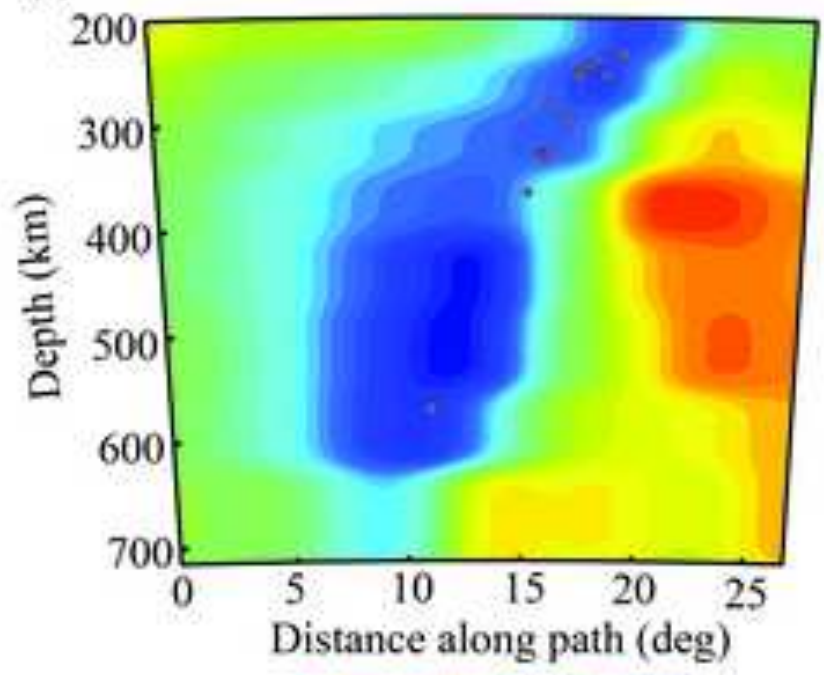

$$
\begin{aligned}
& \begin{array}{lll}
-1 & 0 & 1
\end{array} \\
& \delta \mathrm{Vp} / \mathrm{Vp}(\%)
\end{aligned}
$$


(a)

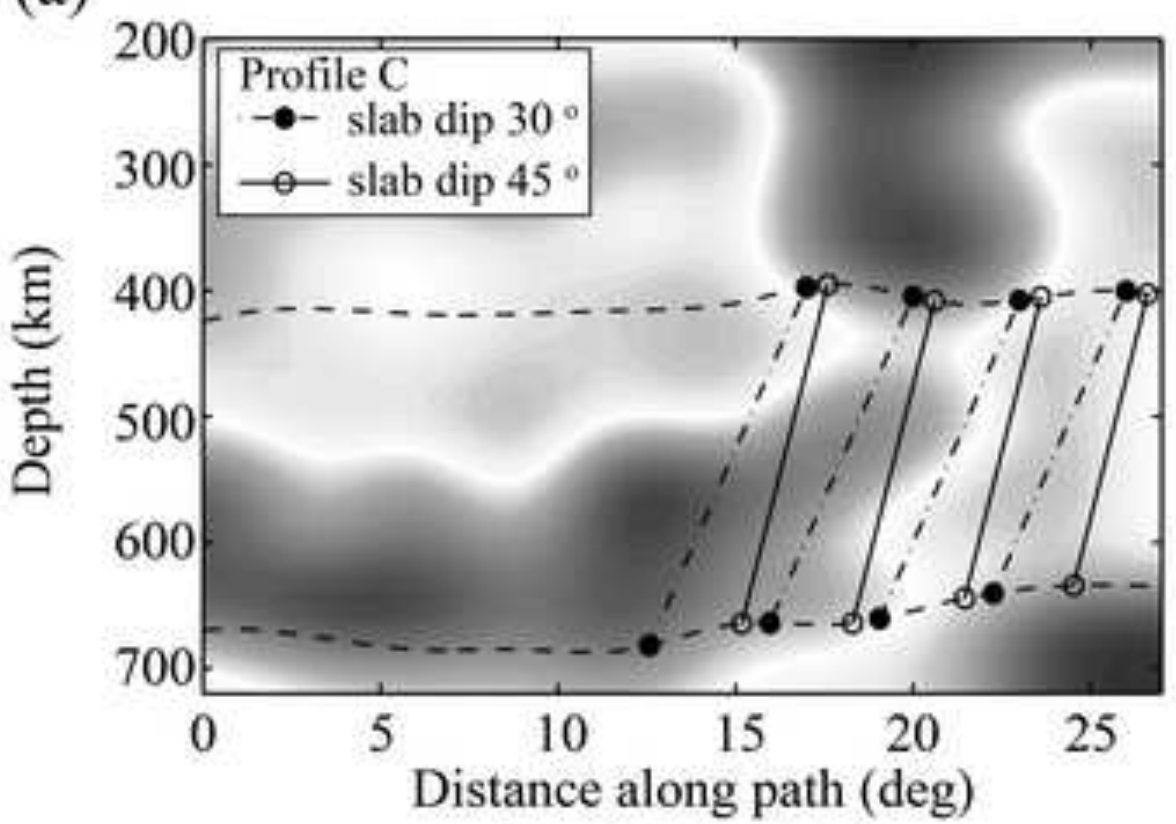

(b)

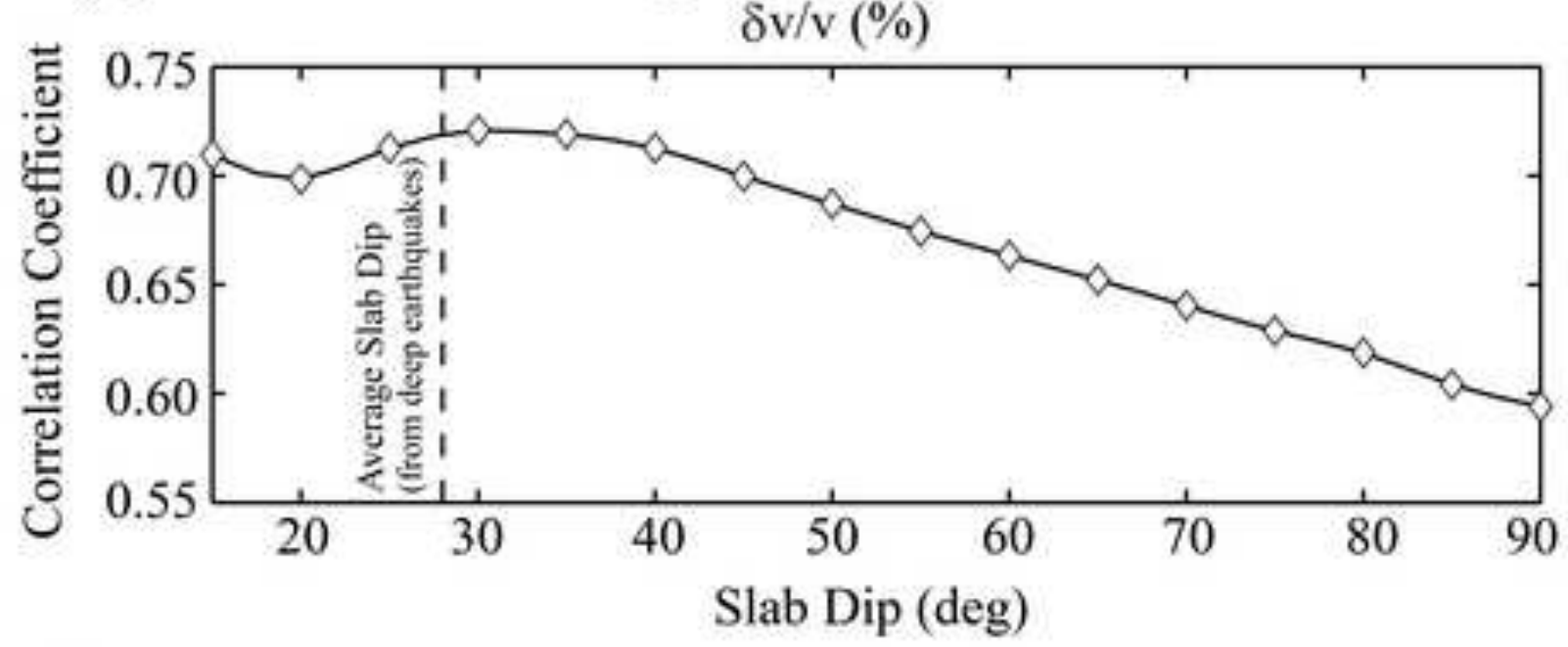

(c)

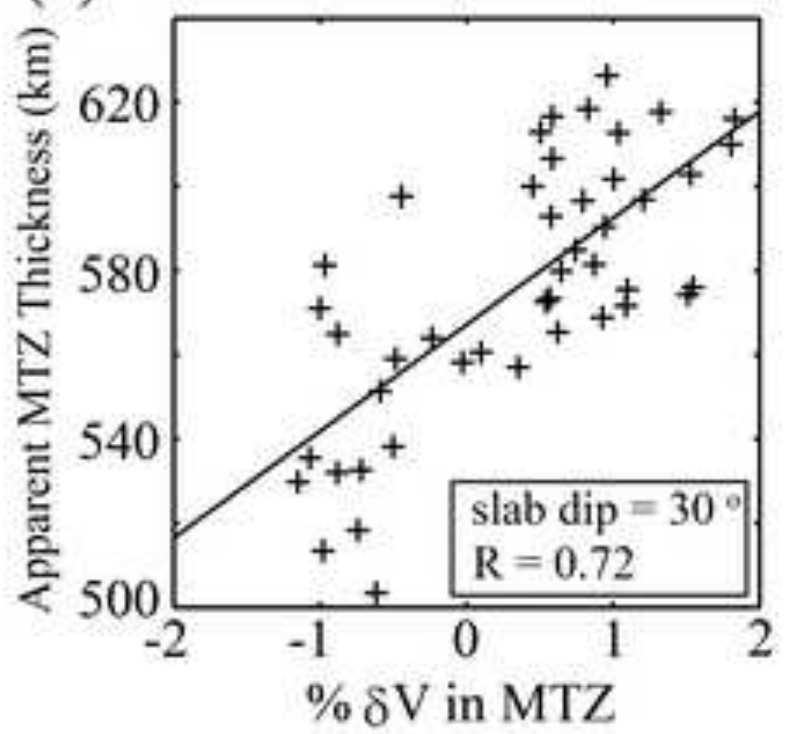

(d)

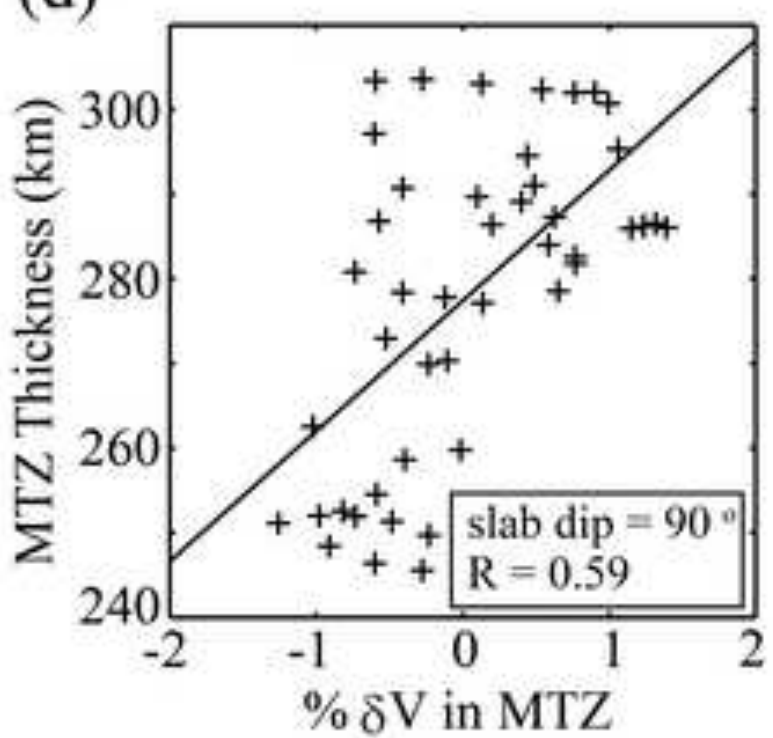



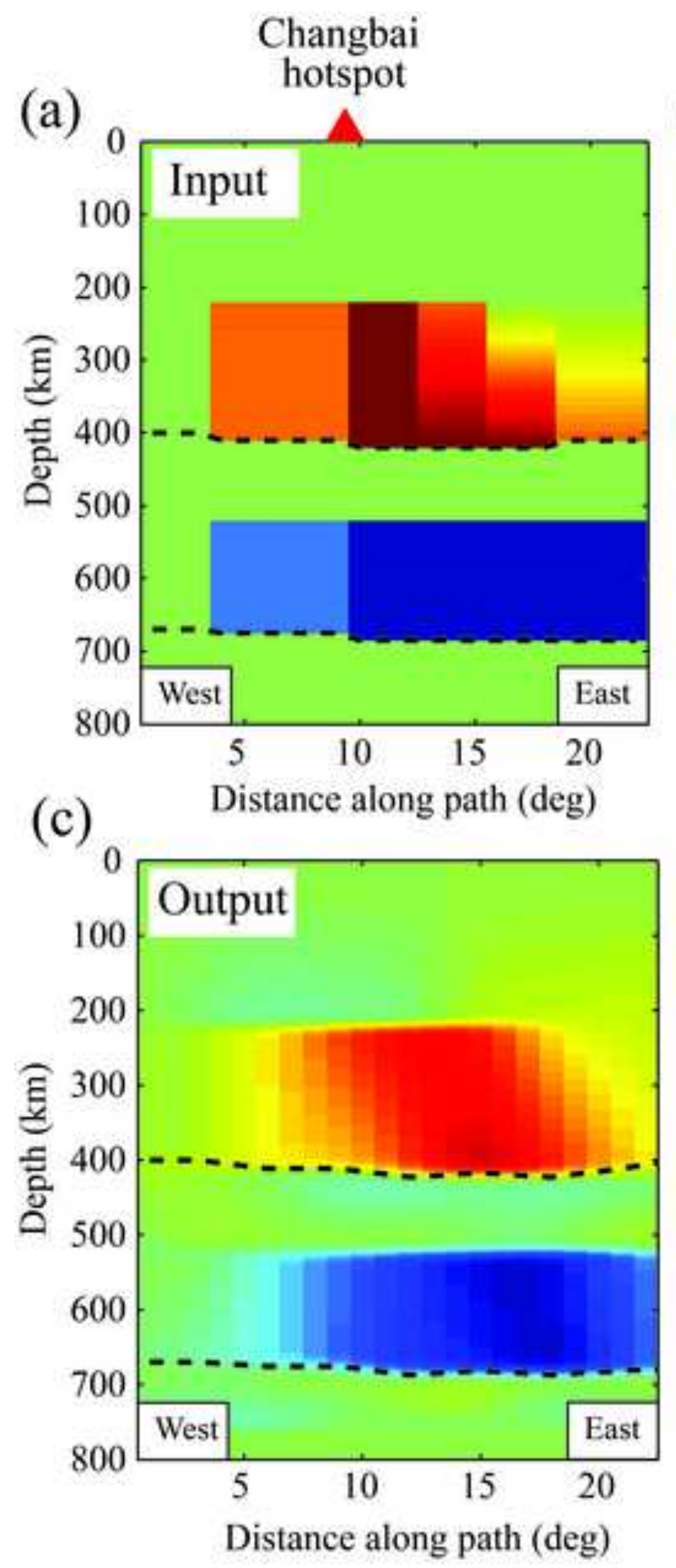

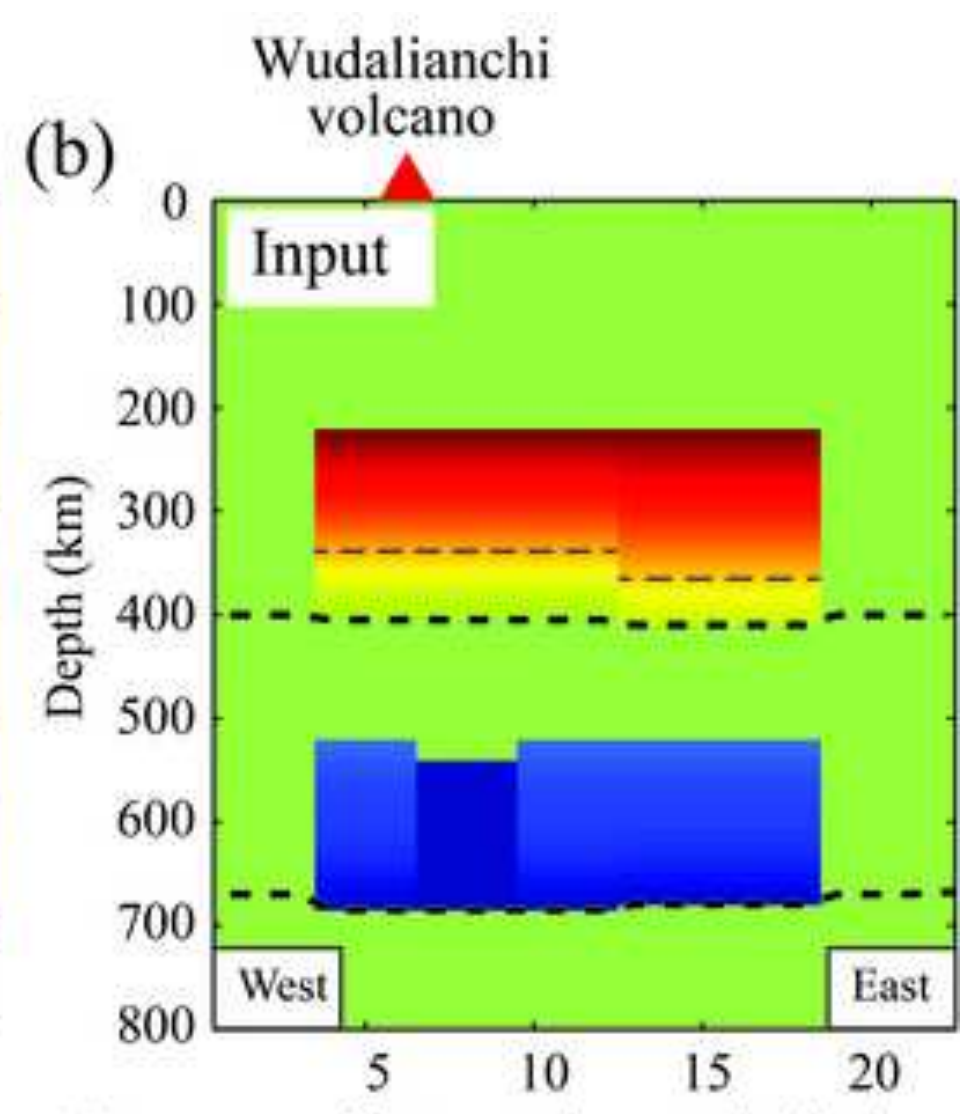

(d)

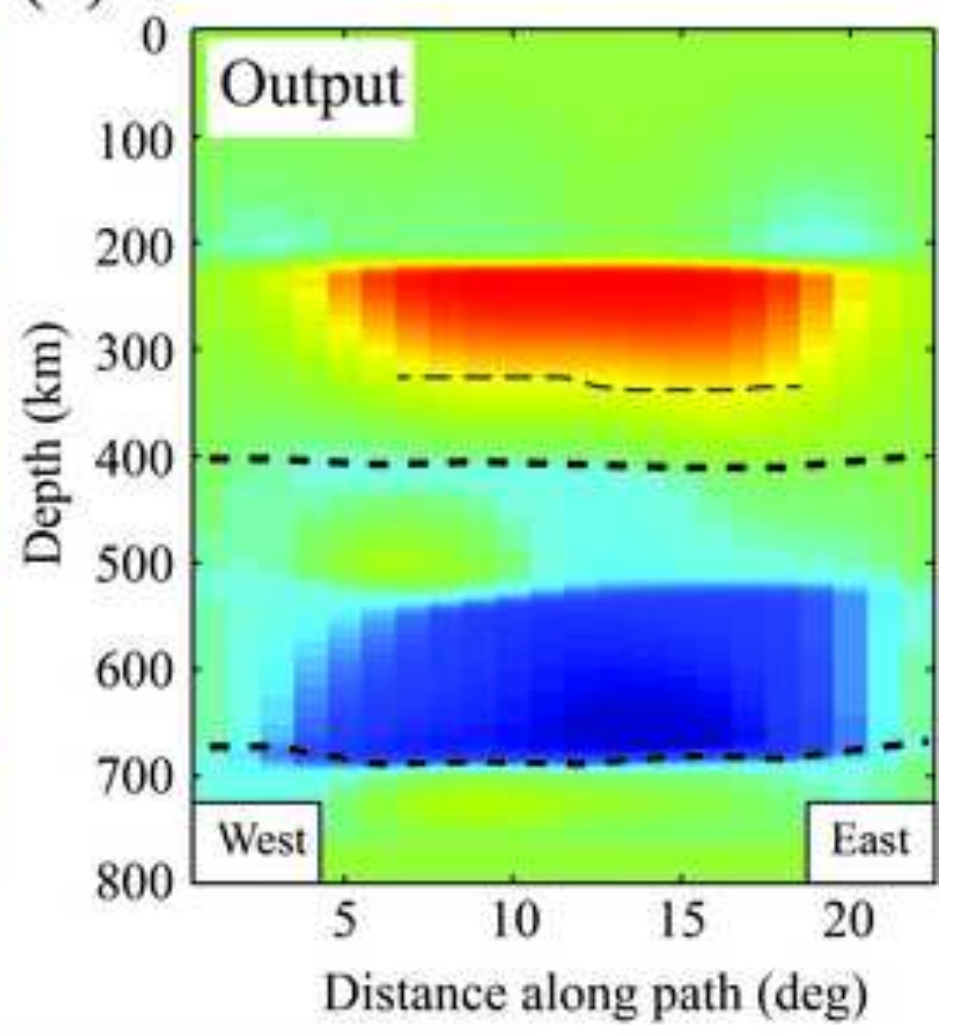

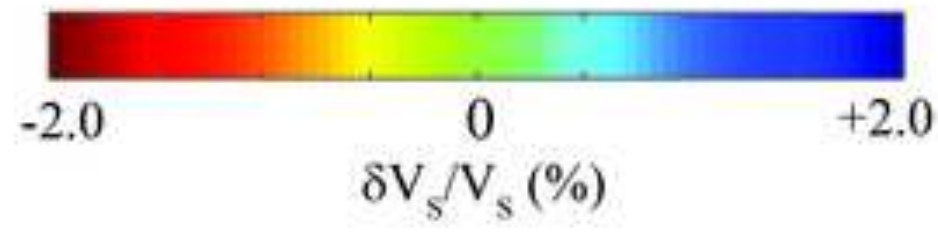


(a)

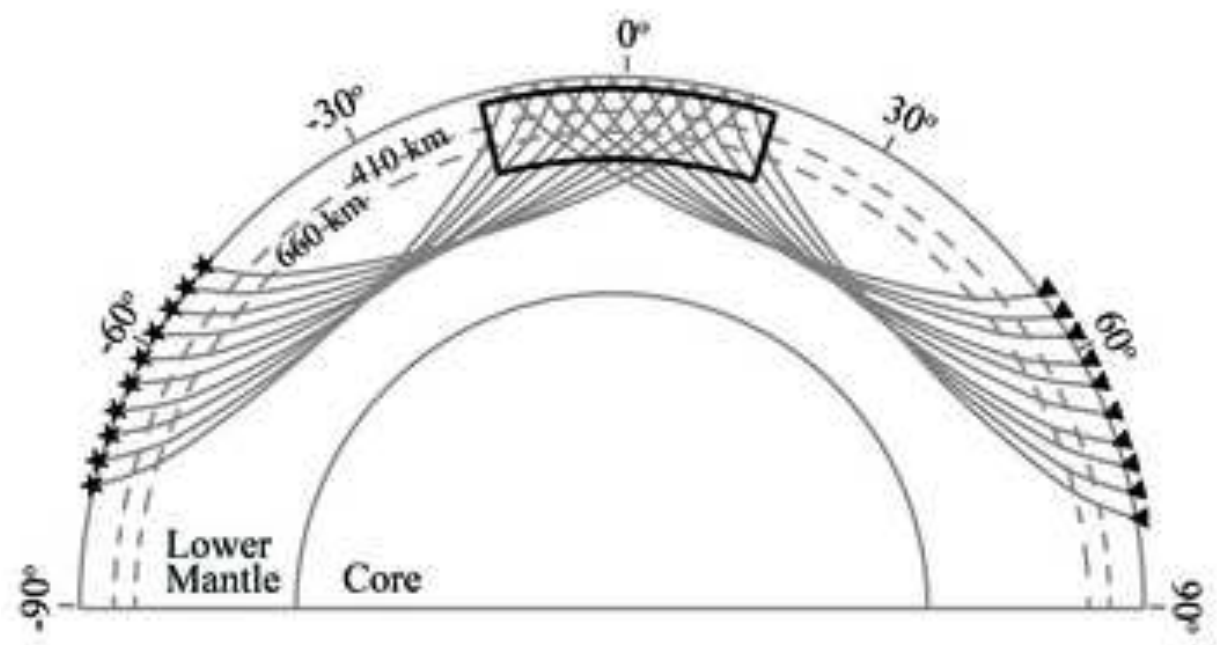

(c)

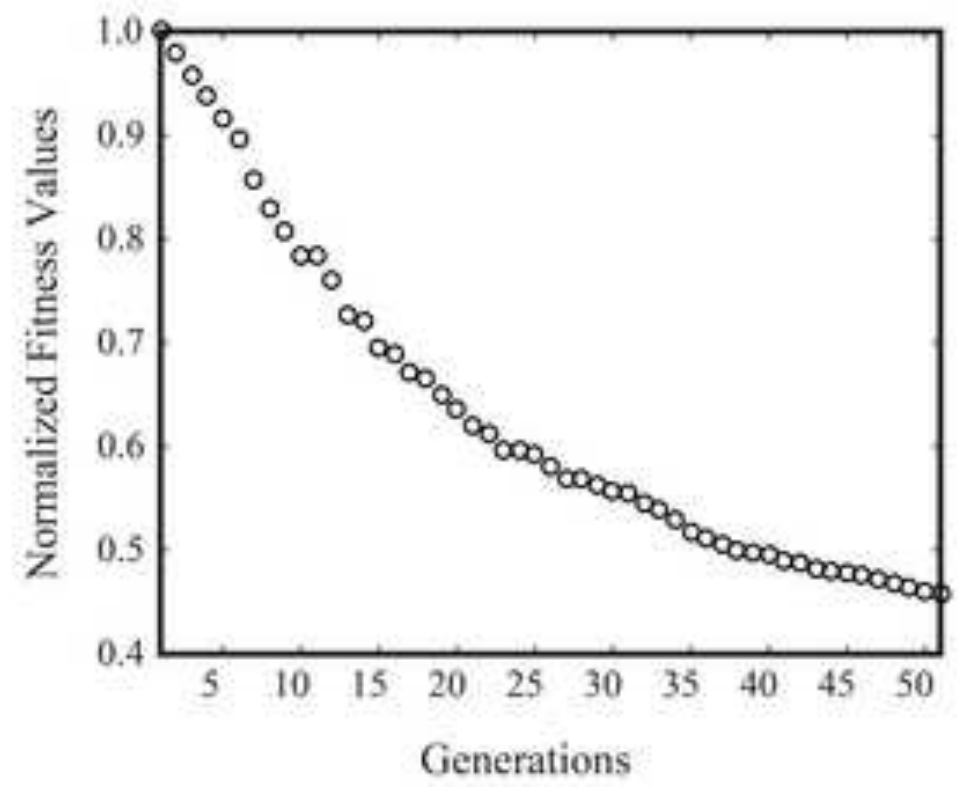

(b)

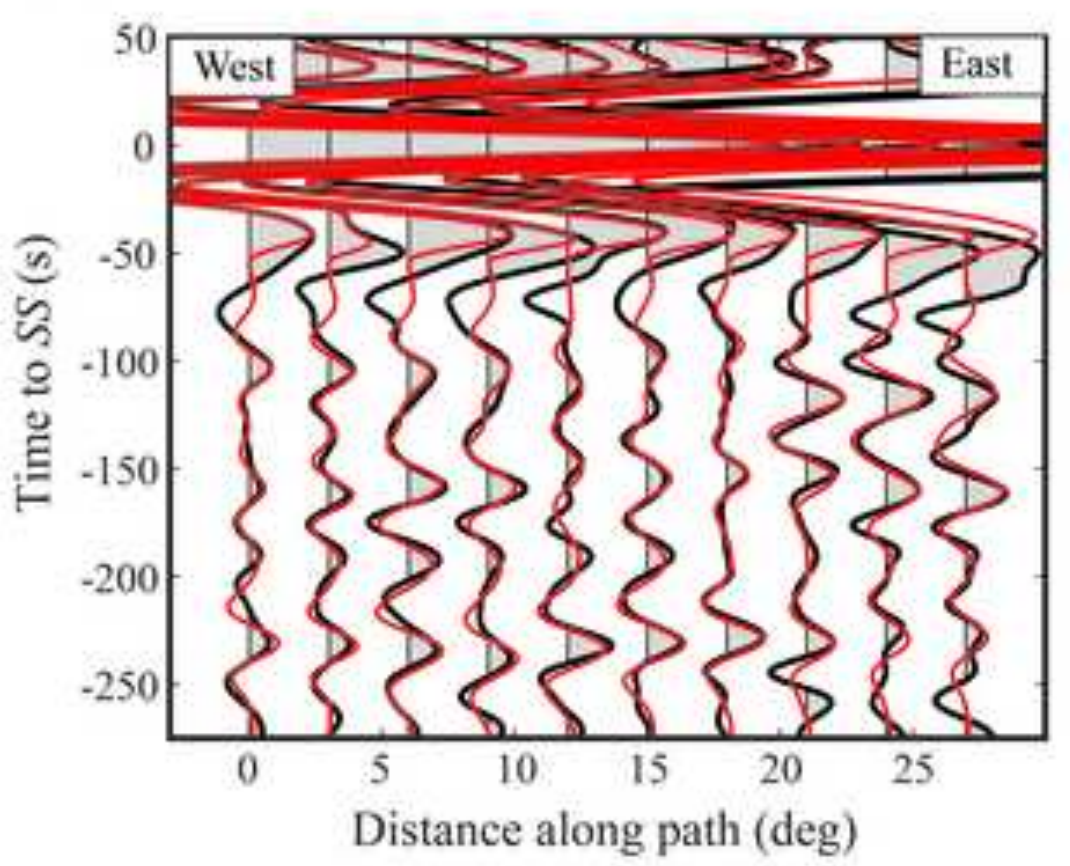

(d)

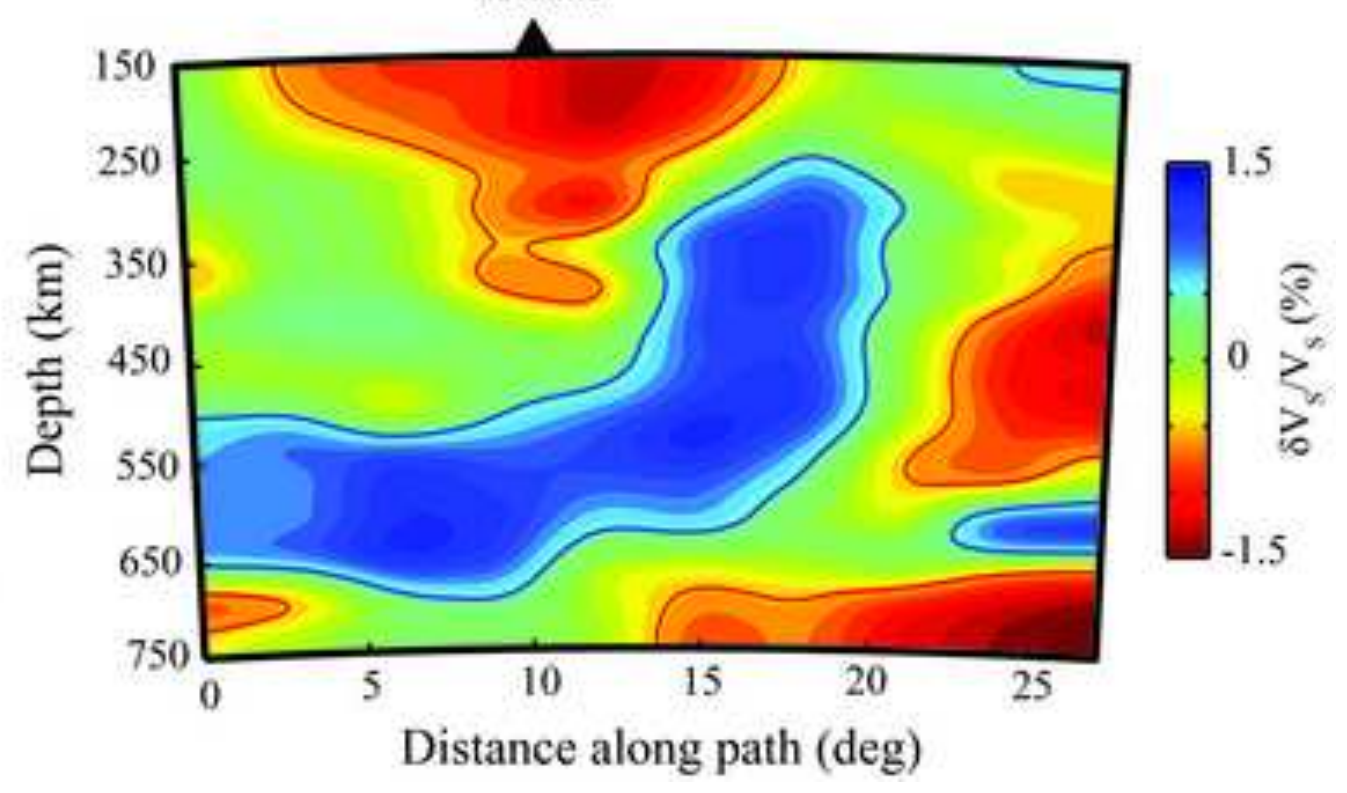



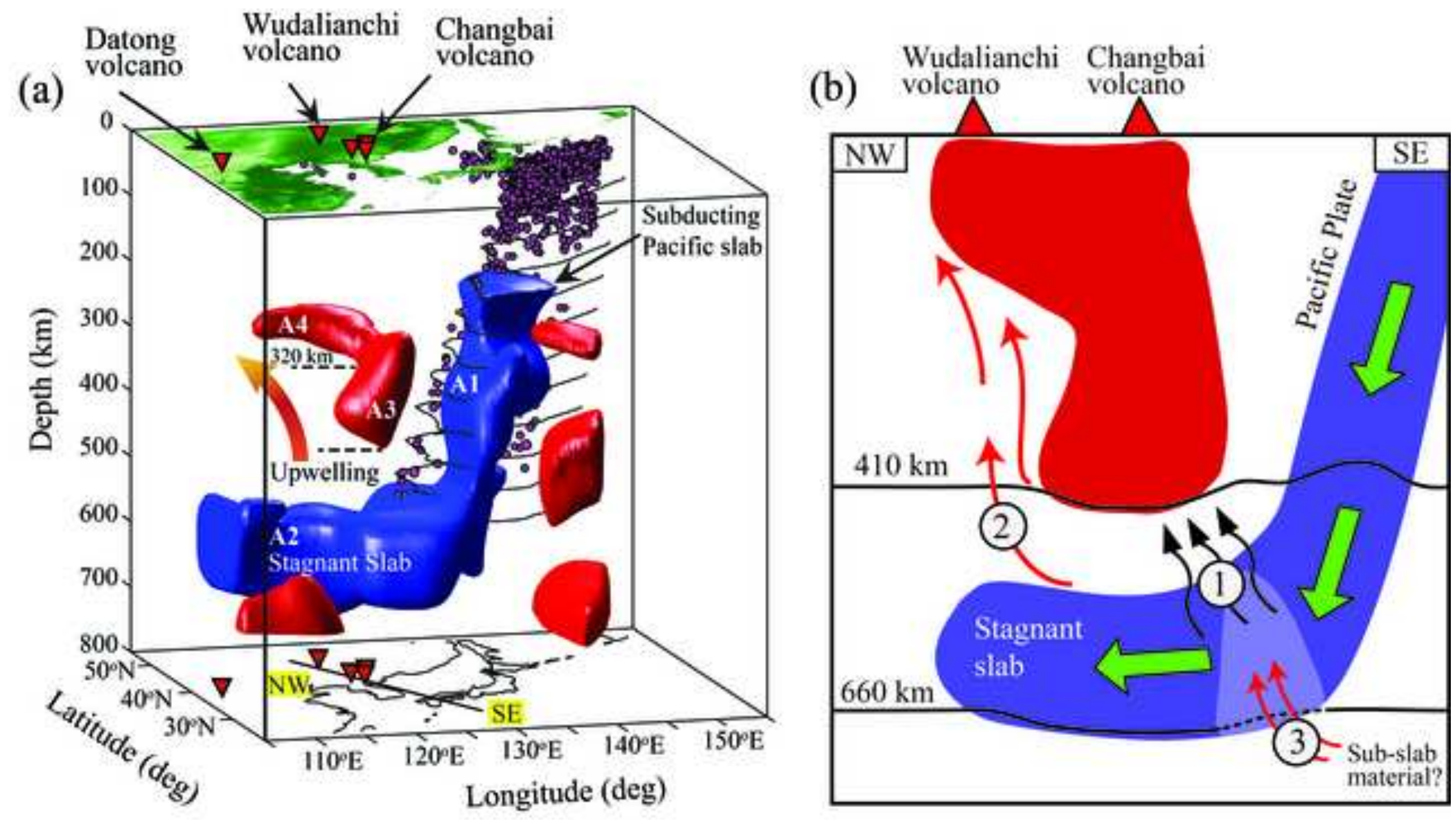